\title{
Electromagnetic dipole moment and time reversal invariance violating interactions of high energy short-lived particles in bent and straight crystals
}

\author{
V. G. Baryshevsky $\odot$ \\ Research Institute for Nuclear Problems, Belarusian State University, \\ 11 Bobruiskaya street, 220030, Minsk, Belarus
}

(Received 5 December 2018; published 27 August 2019)

\begin{abstract}
A particle, which moves in a crystal experiences weak interactions with electrons and nuclei along with electromagnetic interaction. Measuring the polarization vector and the angular distribution of charged and neutral particles scattered by axes (planes) of an unbent (straight) crystal enables to obtain restrictions for the electromagnetic dipole moments value and for magnitudes of constants describing T-odd ( $C P$-odd) interactions beyond the standard model. Spin precession of channeled particles in bent crystals at the LHC gives a unique possibility for measurement of constants determining T-odd ( $C P$-odd) violating interactions of short-lived baryons and $\tau$-leptons with electrons and nuclei (nucleons), similarly to the possibility of measuring particles' electric and magnetic moments. Methods to separate P-odd spin rotation from the MDM-caused and T-odd spin rotations are discussed.
\end{abstract}

DOI: 10.1103/PhysRevAccelBeams.22.081004

\section{INTRODUCTION}

Violation of parity (P) and time reversal (T) symmetries lead to appearance of numerous processes allowing investigation of physics beyond the standard model. Recently, the experimental approach was proposed [1-4] to search for the electromagnetic dipole moments (EDM) of charged short-lived heavy baryons and $\tau$-leptons using bent crystals at LHC. According to [5-7] the same approach gives a unique possibility for investigation of P-odd T-even and P-odd T-odd ( $C P$-odd) interactions of short-lived baryons ( $\tau$-leptons) with electrons and nuclei. Constraints on constants of the above interactions can also be obtained.

This paper demonstrates that measuring the polarization vector and the angular distribution of charged and neutral particles scattered by axes (planes) of an unbent (straight) crystal enables to obtain restrictions for the EDM value and for magnitudes of constants describing T-odd ( $C P$-odd) interactions beyond the standard model.

This paper also considers how P- and T-odd effects, which accompany interaction of baryons ( $\tau$-leptons) with electrons and nuclei in crystals, influence EDM measurement at the LHC. Experimental methods to distinguish different contributions to spin rotation are suggested.

Published by the American Physical Society under the terms of the Creative Commons Attribution 4.0 International license. Further distribution of this work must maintain attribution to the author(s) and the published article's title, journal citation, and DOI.

\section{RELATIVISTIC PARTICLE SPIN INTERACTIONS WITH CRYSTALS}

Since the motion of a high energy particle in a crystal is of quasiclassical nature, the evolution of the particle spin in the electromagnetic fields inside the crystal is governed by the Thomas-Bargmann-Michel-Telegdi (T-BMT) equations [8]. The T-BMT equation describes spin motion in the rest frame of the particle, wherein spin is described by three component vector $\vec{S}$. In practice the T-BMT equation well describes the spin precession in external electric and magnetic fields encountered in typical present accelerators. Study of the T-BMT equation enables one to determine the major peculiarities of spin motion in an external electromagnetic field, to describe the spin rotation effect for particles in a crystal and to apply it for measuring magnetic moments of unstable particles [2,9-17]. However, it should be taken into account that particles in an accelerator or a bent crystal have energy spread and move along different orbits. This necessitates to average the spin-dependent parameters of the particle over phase space of the particle beam. That is why one should always bear in mind the distinction between beam polarization $\vec{\xi}$ and spin vector $\vec{S}$. A complete description of particle spin motion can be made by the use of the spin density matrices equation (for more details, see $[10,18]$ ). For the case of ultrarelativistic baryons with spin $S=1 / 2$ the T-BMT equations supplied with the term, which is responsible for interaction between particle EDM and the electric field, can be written as follows $(\gamma \gg 1, \gamma$ is the Lorentz factor) $[1,2,19,20]$ : 


$$
\frac{d \vec{\xi}}{d t}=\left[\vec{\xi} \times \vec{\Omega}_{\mathrm{magn}}\right]+\left[\vec{\xi} \times \vec{\Omega}_{\mathrm{EDM}}\right]
$$

where $\vec{\xi}$ is the particle polarization vector, $\vec{\Omega}_{\text {magn }}=$ $-\frac{e(g-2)}{2 m c}\left[\vec{\beta} \times \vec{E}_{\perp}\right], g$ is the gyromagnetic ratio (by definition, the particle magnetic moment $\mu=\frac{e g \hbar}{2 m c} S$, where $S$ is the particle spin), $\vec{\Omega}_{\mathrm{EDM}}=\frac{2 e d}{\hbar} \vec{E}_{\perp}, \vec{E}_{\perp}$ is an electric field component perpendicular to the particle velocity $\vec{v}$, the unit vector $\vec{\beta}$ is parallel to the velocity $\vec{v}$, and quantity $D=e d$ is the electric dipole moment.

Note that the authors of [3] use for electric dipole moment the following expression: $\vec{\delta}=J d \mu_{B} \vec{s}$, where $J$ is the particle spin, $d$ is the dimensionless factor referred to as the gyroelectric ratio, $\mu_{B}=\frac{e \hbar}{2 m c}$ is the particle magneton, and $\vec{s}$ is the spin polarization ratio.

It should be mentioned that for particles with spin $3 / 2$ ( $\Omega^{ \pm}$hyperon) T-BMT equations should be supplemented by the terms which consider possession of electric quadrupole moment by the particle $[10,11]$. Moreover, the $\Omega^{ \pm}$ hyperon could also possess the T-odd magnetic quadrupole moment, because its spin value is as high as $3 / 2$. According to $[10,11]$ investigation of spin rotation for $\Omega^{ \pm}$hyperons in straight and bent crystals enables to measure the quadrupole moment of the $\Omega^{ \pm}$hyperon, which cannot be measured by the use of available in a laboratory noncrystalline macroscopic nonuniform electric fields.

\section{P- AND T-ODD SPIN INTERACTIONS IN CRYSTALS}

The general expression for the amplitude of elastic coherent scattering of a spin $1 / 2$ particle by a spinless (unpolarized) atom in the presence of electromagnetic, strong and $P_{-}, T$-odd weak interactions can be written as

$$
\hat{F}(\vec{q})=A(\vec{q})+B(\vec{q}) \vec{\sigma} \vec{N}+B_{w}(\vec{q}) \vec{\sigma} \vec{N}_{w}+B_{T} \vec{\sigma} \vec{N}_{T},
$$

where $A(\vec{q})$ is the spin-independent part of scattering amplitude, which is caused by electromagnetic, strong and weak interactions of the particle with electrons and nucleus of the atom, $\hbar \vec{q}=\hbar \vec{k}^{\prime}-\hbar \vec{k}$ is the transmitted momentum, $\hbar \vec{k}^{\prime}$ is the momentum of the scattered particle, $\hbar \vec{k}$ is the momentum of the incident baryon, $\vec{k}^{\prime}$ and $\vec{k}$ are the wave vectors, $\vec{N}=\frac{\left[\vec{k}^{\prime} \times \vec{k}\right]}{\left[\vec{k}^{\prime} \times \vec{k}\right]}, N_{w}=\frac{\vec{k}^{\prime}+\vec{k}}{\left|\vec{k}^{\prime}+\vec{k}\right|}, \vec{N}_{T}=\frac{\vec{k}^{\prime}-\vec{k}}{\left|\vec{k}^{\prime}-\vec{k}\right|}, \vec{\sigma}=$ $\left(\sigma_{x}, \sigma_{y}, \sigma_{z}\right)$ are the Pauli matrices.

The term, which is proportional to $\vec{\sigma} \vec{N}$, is responsible for the contribution to the scattering process, which is caused by spin-orbit interaction.

The spin-orbit electromagnetic interaction is determined by the particle magnetic moment. The P-odd T-even part of the scattering amplitude (it is proportional to $\vec{\sigma} \vec{N}_{W}$ ) is determined by P-odd T-even interactions of baryon
( $\tau$-lepton) with electrons and nuclei. The T-odd part of the scattering amplitude (it is proportional to $\vec{\sigma} \vec{N}_{T}$ ) is determined by the electric dipole moment and short-range particle-electron and particle-nucleus T-odd interactions. The measurement of the amplitude $B_{T}$ enables the study of physics beyond the standard model and getting limits for the constants, which determine such interactions in hadron and lepton sectors.

The amplitude $\hat{F}(\vec{q})$ determines the cross section of particle scattering by a crystal and polarization vector of the scattered particle. Let us now consider a thin crystal, for which effects caused by channeling are not essential. The scattering cross section for a thin crystal can be written as [11]

$\frac{d \sigma_{c r}}{d \Omega}=\frac{d \sigma}{d \Omega}\left\{\left(1-e^{-\overline{u^{2}} q^{2}}\right)+\frac{1}{N}\left|\sum_{n} e^{i \vec{q} \vec{r}_{n}^{0}}\right|^{2} e^{-\bar{u}^{2} q^{2}}\right\}$,

where $\vec{r}_{n}^{0}$ is the coordinate of the center of gravity of the crystal nucleus, and $\overline{u^{2}}$ is the mean square of thermal oscillations of nuclei in the crystal. The first term describes incoherent scattering, caused by the thermal vibration of crystal nuclei and the second one describes the coherent scattering due to periodic arrangement of crystal nuclei (atoms).

Quantity $\frac{d \sigma}{d \Omega}$ describes the cross section of baryon scattering by atoms of the crystal:

$$
\frac{d \sigma}{d \Omega}=\operatorname{tr} \hat{\rho} \hat{F}^{+}(\vec{q}) \hat{F}(\vec{q})
$$

where $\hat{\rho}$ is the spin density matrix of the incident particle.

The polarization vector of the particle, which has undergone a single scattering event, can be found using the following expression:

$$
\vec{\xi}=\frac{\operatorname{tr} \hat{\rho} \hat{F}^{+} \vec{\sigma} \hat{F}}{\operatorname{tr} \hat{\rho} \hat{F}^{+} \hat{F}}=\frac{\operatorname{tr} \hat{\rho} \hat{F}^{+} \vec{\sigma} \hat{F}}{\frac{d \sigma}{d \Omega}} .
$$

Using (2) one can obtain the following expressions for polarization vector of the scattered particle [7]:

$$
\vec{\xi}=\vec{\xi}_{\text {so }}+\vec{\xi}_{w}+\vec{\xi}_{T}
$$

where $\vec{\xi}_{\text {so }}$ is the contribution to polarization vector due to spin-orbit interaction, $\vec{\xi}_{w}$ is that due to weak parity violating interaction, and $\vec{\xi}_{T}$ is the contribution caused by $T$-odd interaction:

$$
\begin{aligned}
\vec{\xi}_{\mathrm{so}}= & \left\{\left(|A|^{2}-|B|^{2}\right) \vec{\xi}_{0}+2|B|^{2} \vec{N}\left(\vec{N} \cdot \vec{\xi}_{0}\right)\right. \\
& \left.+2 \operatorname{Im}\left(A B^{*}\right)\left[\vec{N} \times \vec{\xi}_{0}\right]+2 \vec{N} \operatorname{Re}\left(A B^{*}\right)\right\} \cdot\left(\frac{d \sigma}{d \Omega}\right)^{-1}
\end{aligned}
$$




$$
\begin{aligned}
\vec{\xi}_{w}= & \left\{\left(|A|^{2}-\left|B_{w}\right|^{2}\right) \vec{\xi}_{0}+2\left|B_{w}\right|^{2} \vec{N}_{w}\left(\vec{N}_{w} \cdot \vec{\xi}_{0}\right)\right. \\
& \left.+2 \operatorname{Im}\left(A B_{w}^{*}\right)\left[\vec{N}_{w} \times \vec{\xi}_{0}\right]+2 \vec{N}_{w} \operatorname{Re}\left(A B_{w}^{*}\right)\right\} \cdot\left(\frac{d \sigma}{d \Omega}\right)^{-1},
\end{aligned}
$$

$$
\begin{aligned}
\vec{\xi}_{T}= & \left\{\left(|A|^{2}-\left|B_{T}\right|^{2}\right) \vec{\xi}_{0}+2\left|B_{T}\right|^{2} \vec{N}_{T}\left(\vec{N}_{T} \cdot \vec{\xi}_{0}\right)\right. \\
& \left.+2 \operatorname{Im}\left(A B_{T}^{*}\right)\left[\vec{N}_{T} \times \vec{\xi}_{0}\right]+2 \vec{N}_{T} \operatorname{Re}\left(A B_{T}^{*}\right)\right\} \cdot\left(\frac{d \sigma}{d \Omega}\right)^{-1}
\end{aligned}
$$

where $\vec{\xi}_{0}$ is the polarization vector of a particle incident on a target.

The differential cross section in the same case reads as follows:

$$
\begin{aligned}
\frac{d \sigma}{d \Omega}= & \operatorname{tr} \rho F^{+} F \\
= & |A|^{2}+|B|^{2}+\left|B_{w}\right|^{2}+\left|B_{T}\right|^{2}+2 \operatorname{Re}\left(A B^{*}\right) \vec{N} \cdot \vec{\xi}_{0} \\
& +2 \operatorname{Re}\left(A B_{w}^{*}\right) \vec{N}_{w} \cdot \vec{\xi}_{0}+2 \operatorname{Re}\left(A B_{T}^{*}\right) \vec{N}_{T} \cdot \vec{\xi}_{0} .
\end{aligned}
$$

While deriving expressions (7)-(9) and (10) the small terms containing productions $B B_{T}, B B_{w}$ and $B_{w} B_{T}$, which describe interference between spin-orbit P-odd T-even and P-odd T-odd interactions, are omitted.

These terms are much smaller compared to those proportional to productions $A B_{w}$ and $A B_{T}$, which describe interference of weak interaction with strong and electromagnetic interactions.

However, the omitted contributions could be significant for neutral particles (see comments hereafter).

In case of neutral particles there is no Coulomb scattering, therefore, the terms proportional to $B B_{w}$ and $B B_{T}$ could also significantly contribute to anisotropy and spin rotation. In this case expression (8) for $\xi_{w}$ should be appended with addition as follows:

$$
\begin{aligned}
\Delta \xi_{w}= & \left\{2 \operatorname{Re}\left(B^{*} B_{w}\right)\left[\left(\overrightarrow{\xi_{0}} \vec{N}\right) \vec{N}_{w}+\left(\vec{\xi}_{0} \vec{N}_{w}\right) \vec{N}\right]\right. \\
& \left.+2 \operatorname{Im}\left(B^{*} B_{w}\right)\left[\vec{N} \times \vec{N}_{w}\right]\right\} \frac{d \sigma^{-1}}{d \Omega} .
\end{aligned}
$$

Expression (9) for $\xi_{T}$ should be appended with the following summand:

$$
\begin{aligned}
\Delta \xi_{T}= & \left\{2 \operatorname{Re}\left(B^{*} B_{T}\right)\left[\left(\overrightarrow{\xi_{0}} \vec{N}\right) \vec{N}_{T}+\left(\vec{\xi}_{0} \vec{N}_{T}\right) \vec{N}\right]\right. \\
& \left.+2 \operatorname{Im}\left(B^{*} B_{T}\right)\left[\vec{N} \times \vec{N}_{T}\right]\right\} \frac{d \sigma^{-1}}{d \Omega} .
\end{aligned}
$$

Expression (10) for $\frac{d \sigma}{d \Omega}$ should be appended with summand $\frac{d \sigma_{\text {app }}}{d \Omega}$ as follows:

$$
\begin{aligned}
\frac{d \sigma_{\text {app }}}{d \Omega}= & -2 \operatorname{Im}\left(B^{*} B_{w}\right) \vec{\xi}_{0}\left[\vec{N} \times \vec{N}_{w}\right] \\
& -2 \operatorname{Im}\left(B^{*} B_{T}\right) \overrightarrow{\xi_{0}}\left[\vec{N} \times \vec{N}_{T}\right] .
\end{aligned}
$$

According to (7) the angle of polarization vector rotation for a baryon scattered in a crystal is determined by rotations around three mutually orthogonal directions (see terms proportional to $N, N_{w}, N_{T}$ ). The indicated rotations are determined by electromagnetic, strong and weak P, T-odd interactions. It should also be noted that initially unpolarized particle beam $\left(\xi_{0}=0\right)$ in a crystal acquires polarization directed along one of three vectors $\vec{N}, \vec{N}_{w}, \vec{N}_{T}$, which carries information about all types of interaction too. According to (10) amplitude interference results in asymmetry in scattering caused by orientation of vectors $\vec{N}_{T}, \vec{N}$, $\vec{N}_{w}$ with respect to $\vec{\xi}_{0}, \vec{k}^{\prime}$ and $\vec{k}$. Therefore, the angular distribution of scattered particles intensity is anisotropic. Thus, measurements of the polarization, the rotation angle and the angular distribution of intensity for a particle beam scattered by crystal axes enable to study T-odd interactions of positive (negative) charged and neutral short-lived baryons and $\tau$-leptons. In particular, such measurements allow one to obtain restrictions on electric dipole moment of short-lived particles and other T-odd interactions in hadron and lepton sectors. According to [5-7] the mentioned interactions can be much stronger than those predicted by the standard model. Obtaining experimental restrictions on these interactions is important [21-23].

Computer modeling is essential for further analysis. Note that analyzing the angle of rotation and the angular distribution one should consider trajectories of the scattered particles with azimuth angles, which are in the vicinity $\varphi$ and $\varphi+\pi$ (the $z$ axis is directed along the momentum of the incident particle). For such particles contributions to spin rotation caused by EDM (T-odd interaction) have opposite signs. As a result the T-odd spin rotation can be observed in unbent crystal if we use subtraction of the measurement results for angle ranges $\varphi$ and $\varphi+\pi$ from each other. Such a procedure leads to summation of contributions from T-odd rotation. Simultaneous measurement of spin orientation for all $\varphi$ values (as well as for all polar angles) provides intensity increase and, therefore, increase in the sensitivity of the EDM/MDM measurement. Note that use of a crystal with polarized nuclei for measuring polarization of scattered particles also could increase experiment sensitivity. According to (10)-(12) the scattered initially nonpolarized particles acquire polarization, the value of which is proportional to the amplitudes determined by the considered interactions. Thus, use of nonpolarized incident beam results in increase of intensity of scattered polarized particles and, therefore, also enables enhancement of experiment sensitivity.

Let us now evaluate the described effects starting from estimation of anisotropy of the angular distribution of 
scattered particles. According to (10) the anisotropy value is determined by interference of amplitude $A$ with amplitudes $B, B_{w}$ and $B_{T}$. The respective contributions to the intensity of scattered particles associated with amplitudes' interference are given by the following ratios:

$$
\begin{aligned}
G & =\frac{2 \operatorname{Re}(A B)}{|A|^{2}}, \\
G_{w} & =\frac{2 \operatorname{Re}\left(A B_{w}^{*}\right)}{|A|^{2}}, \\
G_{T} & =\frac{2 \operatorname{Re}\left(A B_{T}^{*}\right)}{|A|^{2}} .
\end{aligned}
$$

To observe anisotropy related to $G, G_{w}$ or $G_{T}$ the uncertainty in the number $N$ of scattered particles $\delta \approx \frac{1}{\sqrt{N}}$ should be made small. In other words the number of scattered particles should satisfy the conditions as follows:

$$
N>\frac{1}{G^{2}} ; \frac{1}{G_{w}^{2}} ; \frac{1}{G_{T}^{2}} .
$$

Let us now consider expressions (14) and (15) in more detail. The amplitude $A$ is the sum of the amplitudes $A_{\text {coul }}$ and $A_{s}$ caused by Coulomb and strong nuclear scattering, respectively. The amplitude of spin-orbit scattering $B=$ $B_{\text {magn }}+B_{\text {so }}$ is caused by particle magnetic moment interaction $B_{\text {magn }}$ and by strong nuclear spin-orbit interaction $B_{\text {so }}$. Let us start with evaluation of anisotropy $G$ caused by magnetic and strong nuclear spin-orbit interaction:

$G=\frac{2 \operatorname{Re}\left(A_{s} B_{\mathrm{magn}}^{*}+A_{s} B_{\mathrm{so}}^{*}+A_{\text {coul }} B_{\mathrm{magn}}^{*}+A_{\text {coul }} B_{\mathrm{so}}^{*}\right)}{\left|A_{\mathrm{coul}}+A_{s}\right|^{2}}$.

For further analysis let us pay attention to the fact that from results presented in [24] the scattering amplitude of a particle, which possesses magnetic moment, by a Coulomb field can be expressed as follows:

$A=A_{\text {coul }}(\vartheta)+i \frac{1}{2}\left(\frac{g-2}{g} \frac{\gamma^{2}-1}{\gamma}+\frac{\gamma-1}{\gamma}\right) \vartheta A_{\text {coul }}(\vartheta) \vec{\sigma} \vec{N}$.

From (17) the following expression for amplitude $B_{\text {magn }}$ can be obtained:

$$
B_{\text {magn }}=i \frac{1}{2}\left(\frac{g-2}{g} \frac{\gamma^{2}-1}{\gamma}+\frac{\gamma-1}{\gamma}\right) \vartheta A_{\text {coul }}(\vartheta) .
$$

As a result in the case of elastic Coulomb scattering $\operatorname{Re}\left(A_{\text {coul }} B_{\text {magn }}^{*}\right)=0$. Analysis shows that for the contribution to the scattering amplitude caused by the spin-orbit strong interaction one can obtain the expression similar to (18) by using the optical model of a nucleus:

$$
B_{\mathrm{so}}=i \frac{1}{2}\left(\frac{g_{\mathrm{so}}-2}{g} \frac{\gamma^{2}-1}{\gamma}+\frac{\gamma-1}{\gamma}\right) \vartheta A_{s}(\vartheta) .
$$

The quantity $g_{\text {so }}$ introduced in (19) is similar to the magnetic $g$-factor and depends on the particle energy.

From (19) it follows that $\operatorname{Re}\left(A_{s} B_{\mathrm{so}}^{*}\right)=0$. Therefore,

$$
\operatorname{Re}\left(A B^{*}\right) \simeq A_{s}^{\prime \prime} A_{\text {coul }}^{\prime}\left(\frac{g-2}{4}+\frac{g_{\text {so }}-2}{4}\right) \gamma \vartheta,
$$

here $A_{\text {coul }}^{\prime}=\operatorname{Re} A_{\text {coul }}$ and $A_{s}^{\prime \prime}=\operatorname{Im}\left(A_{s}\right)$. The above expression is obtained with consideration of the imaginary part of Coulomb amplitude $A_{\text {coul }}^{\prime \prime}$ to be smaller as compared to its real part $A_{\text {coul }}^{\prime}: A_{\text {coul }}^{\prime \prime}$ is $Z \alpha$ times smaller as compared to $A_{\text {coul }}^{\prime}$ (here $\alpha$ is the fine-structure constant).

To evaluate the imaginary part of amplitude $A_{s}^{\prime \prime}$ of baryon scattering by a nucleus let us use a model of diffraction scattering. As a result in eikonal approximation $A_{s}^{\prime \prime}$ reads as follows:

$$
A_{s}^{\prime \prime}=R_{\mathrm{nuc}} \frac{J_{1}\left(R_{\mathrm{nuc}} k \vartheta\right)}{\vartheta},
$$

where $R_{\text {nuc }}$ is the radius of nucleus, $J_{1}$ is the Bessel function of the first order. From (20) it follows that for scattering angles $\vartheta \leq \frac{1}{k R_{\text {nuc }}}$ the imaginary part of scattering amplitude $A_{s}^{\prime \prime} \approx k R_{\text {nuc }}^{2}$. The real part of Coulomb amplitude $A_{\text {coul }}^{\prime}=\frac{Z \alpha}{k \vartheta^{2}}$ becomes comparable or even greater than $A_{s}^{\prime \prime}$ for scattering angles $\vartheta \leq \frac{\sqrt{Z \alpha}}{k R_{\text {nuc }}}$.

For a baryon with energy $1 \mathrm{TeV}$ the scattering angle is $\vartheta \leq \sqrt{Z \alpha} \times 10^{-5}$. Therefore, in the range of angles, within which $A_{s}^{\prime \prime} \simeq A_{\text {coul }}^{\prime}, \quad$ parameter $\quad G \simeq\left(\frac{g+g_{\mathrm{so}}}{2}-2\right) \gamma \vartheta \simeq$ $\left(\frac{g+g_{\mathrm{so}}}{2}-2\right) \times 10^{-2}$. As a result, to comply inequality $N>\frac{1}{G^{2}}$, the number of scattered particles should be $N \simeq 10^{4}-10^{5}$.

Parameter $g_{\text {so }}$ depends on the energy of the incident particle in contrast to the $g$-factor, which does not at currently present particle energies. This fact makes it possible to distinguish contributions from $g$ and $g_{\text {so }}$ from each other.

The number $N_{\Lambda_{c}^{+}}$of charmed $\Lambda_{c}^{+}$baryons produced by $10^{17}-10^{18}$ protons in a tungsten target can be found using data published in [1] that gives $N_{\Lambda_{c}^{+}} \approx 10^{13}-10^{14}$. These particles, move within angle $\frac{1}{\gamma} \approx 10^{-3}$.

Hereafter, let us consider the incidence of $\Lambda_{c}^{+}$baryons on a target at the angle equal to or greater than the Lindhard angle, which is for $\mathrm{Si}(\mathrm{Ge})$ of order $\vartheta_{L} \approx 6(7) \times 10^{-6} \mathrm{rad}$. Let us consider a range $\Delta \vartheta \sim 10^{-5}$, which amounts to several Lindhard angles. Within this range $\frac{\Delta \vartheta}{\gamma} \sim 10^{-2}$, therefore, the number of $\Lambda_{c}^{+}$baryons within this range is 
$\Delta N_{\Lambda_{c}^{+}} \approx 10^{11}-10^{12}$. Let us now give evaluation for the number of these particles scattered in a crystal of thickness $l=0.1 \mathrm{~cm}$ :

$$
N \approx \frac{d \sigma}{d \Omega} \Delta \Omega N_{a t} l \Delta N_{\Lambda_{c}^{+}} \approx 10^{-2} \Delta N_{\Lambda_{c}^{+}} \approx 10^{9}-10^{10},
$$

here $N_{\text {at }}$ is the number of atoms in $1 \mathrm{~cm}^{3}$ of target. This value complies condition $N>\frac{1}{G^{2}} \approx 10^{4}-10^{5}$ that enables carrying measurement of baryon magnetic moment by analysis of anisotropy of angular distribution of scattered baryons.

Let us now evaluate baryon scattering anisotropy, which is caused by T-odd processes, for example, presence of particle EDM of order $e \cdot d \sim 10^{-17} e \mathrm{~cm}$.

For a particle possessing EDM, which is scattered in an electric field, the following expression for amplitude $B_{T}$ in eikonal approximation can be used:

$$
B_{T}(\vartheta)=i d k \vartheta A_{\text {coul }}=\frac{d}{\lambda_{c}} \gamma \vartheta A_{\text {coul }}(\vartheta),
$$

where $\lambda_{c}=\frac{\hbar}{m c}$ is the Compton wavelength of the particle. For the supposed EDM value amplitude $B_{T}$ appears to be 3 orders smaller as compared to amplitude of magnetic scattering $B_{\text {magn }}$. Therefore, $G_{T} \approx 10^{-3} \mathrm{G}$ and the number of particles required to make anisotropy, which is associated with amplitude $B_{T}$, is higher $10^{6}$ times i.e., required number of particles $N>10^{10}-10^{11}$. Recall that above for the target with high $Z$ and thickness $l=0.1 \mathrm{~cm}$ we have obtained $N \approx 10^{9}-10^{10}$. Therefore, increasing the target thickness to $l=1 \mathrm{~cm}$ (such a target is still quite thin) and optimizing all the experiment parameters one could expect to observe EDM-caused anisotropy and that caused by other T-odd interactions. Such a possibility is important for studying EDM and T-odd interactions of short-lived particles.

Let us now dwell on the possibility to investigate EDM and other T-odd interactions for $\tau$-leptons, which do not undergo strong interactions. The EDM-caused anisotropy for $\tau$-leptons is suppressed, because the contribution to the cross section, which is caused by interference of Coulomb amplitude and amplitude $B_{T}$ defined by (21), is equal to zero. The nonzero summand is due to interference of Coulomb amplitude and T-odd amplitude caused by neutral currents. Restriction for the magnitude of the latter amplitude enables to evaluate the constant of corresponding interaction. The mentioned interaction is now persistently studied for electrons in optical experiments with atoms [23].

Let us now evaluate the angle of spin rotation for a scattered particle, which possesses EDM. According to (9) the additional polarization component, which arises due to rotation around $\vec{N}_{T}$, is determined by the expression as follows:

$$
\Delta \xi_{\text {Trot }}=\frac{2 \operatorname{Im}\left(A B_{T}^{*}\right)}{|A|^{2}} .
$$

Therefore, for a particle scattered in the Coulomb field the angle of spin rotation can be evaluated using (7) as follows:

$$
\vartheta_{s} \simeq \Delta \xi_{\mathrm{Trot}} \simeq \frac{d}{\lambda_{c}} \gamma \vartheta
$$

According to (23) the angle of spin rotation grows with growth of scattering angle and $\gamma$ (with scattering angle growth the angles of spin rotation caused by other T-odd interactions also grow). For $d=10^{-17} \mathrm{~cm}$ and $\lambda_{c}=$ $10^{-14} \mathrm{~cm}$ the angle of rotation is $\Delta \xi_{\text {Trot }} \simeq 10^{-3} \gamma \vartheta$, therefore for a particle with $\gamma \sim 10^{3}$ the angle of spin rotation is expected to be as high as the scattering angle: $\vartheta_{s} \simeq$ $\Delta \xi_{\text {Trot }} \simeq \vartheta$. Hence, for $\gamma=10^{3}$ and scattering angle $\vartheta \simeq$ $10^{-4}-10^{-5}$ the angle of spin rotation $\vartheta_{s} \simeq \Delta \xi_{\text {Trot }} \simeq$ $10^{-4}-10^{-5}$. The number of detected particles is $N>\frac{1}{\vartheta_{s}^{2}} \simeq 10^{8}-10^{10}$. Recall that in this case spin rotates around direction $\vec{N}_{T}$, which is determined by the direction of transferred momentum $\vec{q}$.

Investigation of anisotropy and rotation angle caused by amplitude of weak P-odd interaction $B_{w}$, which is determined by neutral P-odd currents, is of interest for shortlived baryons and $\tau$-leptons. Recall that for electrons the neutral currents were observed in two types of experiments: at deep inelastic scattering at SLAC accelerator [25-28] and in optical experiments in Novosibirsk [29].

\section{RELATIVISTIC PARTICLE SPIN ROTATION AND INTERACTIONS IN BENT CRYSTALS}

Let us now consider a particle moving in a bent crystal. Expressions for energy of interaction between a particle and a crystal plane (axis), which are obtained in [5-7], allow us to find the equation describing evolution of the particle polarization vector in a bent crystal. The mentioned equations differ from those describing spin evolution in external electromagnetic fields in vacuum by the presence of terms which define contributions to spin rotation from $\mathrm{P}$ and $\mathrm{T}(C P)$ noninvariant interactions between electrons and nuclei. As a result, when carrying the experiments for search the EDM of short-lived baryons and $\tau$-leptons in a bent crystal in addition one can also obtain restrictions for constants of T-odd interactions in hadron and lepton sectors. Moreover, a new effect, which is caused by nonelastic processes, arises: along with the spin precession around vectors $\vec{N}_{m}, \vec{N}_{T}, \vec{\beta}$, the spin components directed along vectors $\vec{N}_{m},\left(\vec{N}_{T}, \vec{\beta}\right)$ appear and, thus, spin dichroism occurs. Let us remind that $\vec{N}_{m}=\left[\vec{\beta} \times \vec{n}_{x}\right], \vec{N}_{T}=\frac{\vec{k}^{\prime}-\vec{k}}{\left|\vec{k}^{\prime}-\vec{k}\right|}$, the unit vector $\vec{\beta}$ is parallel to the velocity $\vec{v}$. Let us elucidate the effect of dichroism on the example of P-odd spin rotation and dichroism. 
Let us note that as scattering amplitude $\hat{F}$ is a complex value, so potential energy $\hat{U}$ is also a complex value. The real part of this energy describes changes in particle energy as a result of interaction with matter, the imaginary part describes absorption.

Every spin-dependent contribution to $\hat{U}$ is of the following structure:

$$
\hat{A}=-(\alpha+i \beta) \vec{\sigma} \vec{N} .
$$

Let us compare this expression with that for energy of the interaction between the magnetic moment $\vec{\mu}$ and the magnetic field $\vec{B}$ :

$$
\hat{U}_{\mathrm{magn}}=-\mu \vec{\sigma} \vec{B}
$$

It can be seen that terms proportional to $\alpha$ in $\hat{A}$ cause spin rotation around $\vec{N}$. The imaginary part shows that absorption in matter depends on spin orientation regarding $\vec{N}$. As a result the spin component directed along $\vec{N}$ can appear (spin dichroism occurs [10]).

The analogy between (24) and (25) leads us to the conclusion that a particle in matter is affected by pseudomagnetic fields caused by strong and weak interactions (for low energy neutrons the effects determined by such fields were discovered and have been investigated for many years, see [10]). Let us consider now baryons with polarization vector oriented at a certain angle to the direction of $\vec{\beta}$ be incident on medium. This baryon state can be considered as a superposition of two states with polarizations along and opposite to the momentum direction defined by unit vector $\vec{\beta}$. The wave function of a particle before entering the target reads as

$$
\psi(\vec{r})=e^{i \vec{k} \vec{r}} \chi_{n}, \chi_{n}=\left(\begin{array}{c}
c_{1} \\
c_{2}
\end{array}\right)
$$

or

$$
\psi(\vec{r})=c_{1} e^{i \vec{k} \vec{r}}\left(\begin{array}{l}
1 \\
0
\end{array}\right)+c_{2} e^{i \vec{k} \vec{r}}\left(\begin{array}{l}
0 \\
1
\end{array}\right)
$$

Suppose the direction $\vec{\beta}$ is along the $z$ axis. The coefficients $c_{1}$ and $c_{2}$ in polar coordinates are defined in $[8,10]$ as follows:

$$
\begin{aligned}
& c_{1}=\left( \pm e^{-i \varphi / 2} \cos \frac{\vartheta}{2}\right) \\
& c_{1}=\left(e^{i \varphi / 2} \sin \frac{\vartheta}{2}\right)
\end{aligned}
$$

where $\vartheta$ and $\varphi$ are the polar and azimuthal angles. The state $\left(\begin{array}{l}1 \\ 0\end{array}\right)$ has the refractive index $n_{+}$, while state of type $\left(\begin{array}{l}0 \\ 1\end{array}\right)$ has the refractive index $n_{-}$. If a baryon with spin parallel to vector $\vec{\beta}$ [spin state $\left(\begin{array}{l}1 \\ 0\end{array}\right)$ ] is incident on the target, its motion in matter can be described by wave function $\psi_{+}(r)=$

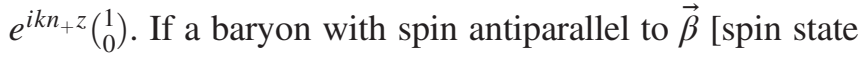
$\left(\begin{array}{l}0 \\ 1\end{array}\right)$ ] is incident on the target, then in matter it is described by the wave function $\psi_{-}(r)=e^{i k n_{-} z}\left(\begin{array}{l}0 \\ 1\end{array}\right)$. If a baryon with an arbitrary spin direction falls on the target, its wave function [see (27)] is the superposition of states $\left(\begin{array}{l}1 \\ 0\end{array}\right)$ and $\left(\begin{array}{l}0 \\ 1\end{array}\right)$. As a consequence, the wave function of a baryon in matter is also the superposition of these states, and can be written as

$\psi(\vec{r})=\left(\begin{array}{lll}c_{1} & \psi_{+} & (\vec{r}) \\ c_{2} & \psi_{-} & (\vec{r})\end{array}\right)=c_{1} e^{i k n_{+} z}\left(\begin{array}{l}1 \\ 0\end{array}\right)+c_{2} e^{i k n_{-} z}\left(\begin{array}{l}0 \\ 1\end{array}\right)$.

Now let us consider how baryon polarization changes as they penetrate into the interior of the target (with the growth of the target thickness). Suppose we have a detector that transmits the particles with spin polarized along a certain direction in the detector (the axis of the detector) and absorbs the particles with the opposite spin direction. Such a detector is the analog of the Nicol prism [30] used in optics for analyzing the polarization of light. When polarized light is incident on the Nicol prism, one component of light polarization passes through it, while the component orthogonal to the axis of the Nicol prism is absorbed. In the case of baryons, a target with polarized nuclei may act as a detector. As the scattering cross section of a polarized baryon depends on whether the baryon spin is oriented along the direction of the polarization vector of the nucleus or is opposite to it, so baryon absorption in the detector exhibits the same dependence [10]. Suppose that the detector axis is parallel to the $z$ axis, along which the detector nuclei are polarized. In this case the detector analyzes those components of baryon spin, which are directed along the $z$ axis and in opposite direction. From (28) it follows that the amplitude $A^{(+)}$of probability to find the baryon with spin state $\left(\begin{array}{l}1 \\ 0\end{array}\right)$, i.e., to find the baryon polarized parallel to the $z$ axis, is given by the expression

$$
A^{(+)}=\left(\begin{array}{ll}
1 & 0
\end{array}\right) \psi=c_{1} e^{i k n_{+} z} .
$$

The probability is

$$
P_{z}^{(+)}=\left|\left(\begin{array}{ll}
1 & 0
\end{array}\right) \psi\right|^{2}=\left|c_{1}^{2}\right| e^{-2 k \operatorname{Im}\left(n_{+}\right) z}=\left|c_{1}\right|^{2} e^{-\rho \sigma_{+} z} .
$$

Similarly, the probability $P_{z}^{(-)}$to find the baryon polarized opposite to the $z$ axis is

$$
P_{z}^{(-)}=\left|\left(\begin{array}{ll}
0 & 1
\end{array}\right) \psi\right|^{2}=\left|c_{2}\right|^{2} e^{-2 k \operatorname{Im}\left(n_{-}\right) z}=\left|c_{2}\right|^{2} e^{-\rho \sigma_{-} z},
$$

where $\sigma_{ \pm}$is the total cross section of scattering the baryon polarized parallel (antiparallel) to the baryon momentum by 
the nucleus. Since owing to P-violation in matter $\operatorname{Im}\left(n_{+}\right) \neq$ $\operatorname{Im}\left(n_{-}\right)\left(\sigma_{+} \neq \sigma_{-}\right)$, so one of the components of the baryon spin wave function decays faster and at some depth the rapidly damped component may be neglected. The beam will appear polarized along the $z$ axis (along the direction of the particle momentum $n$ ). Let us now rotate the detector so that its polarization axis becomes perpendicular to the direction $n$. Choose the direction of the polarization axis of the detector as the $x$ axis. Now the detector analyzes those components of the baryon spin, which are directed along and opposite to the $x$ axis. To determine the probability $P_{x}^{( \pm)}$to find the component of the baryon spin parallel (antiparallel) to the direction of the $x$ axis, one should expand the wave function (28) in terms of the spin wave functions $\chi_{x}^{ \pm}$, which are the eigenfunctions of operator $\hat{S}_{x}$ of the spin projection onto the $x$ axis. They have the form

$$
\chi_{x}^{ \pm}=\frac{1}{\sqrt{2}}\left(\begin{array}{c}
1 \\
\pm 1
\end{array}\right)
$$

As a result, we find that probabilities $P_{x}^{( \pm)}$of baryon spin polarization along and opposite to the $x$ axis change with $z$ as

$$
\begin{aligned}
P_{x}^{(+)}= & \frac{1}{2}\left\{\left|c_{2}\right|^{2} e^{-2 k \operatorname{Im}\left(n_{+}\right) z}+\left|c_{2}\right|^{2} e^{-2 k \operatorname{Im}\left(n_{-}\right) z}\right. \\
& \left.+2\left|c_{1}\right| c_{2} \mid e^{-k \operatorname{Im}\left(n_{+}+n_{-}\right) z} \cos \left[k \operatorname{Re}\left(n_{+}-n_{-}\right) z+\delta\right]\right\}, \\
P_{x}^{(-)}= & \frac{1}{2}\left\{\left|c_{1}\right|^{2} e^{-2 k \operatorname{Im}\left(n_{+}\right) z}+\left|c_{2}\right|^{2} e^{-2 k \operatorname{Im}\left(n_{-}\right) z}\right. \\
& \left.-2\left|c_{1}\right| c_{2} \mid e^{-k \operatorname{Im}\left(n_{+}+n_{-}\right) z} \cos \left[k \operatorname{Re}\left(n_{+}-n_{-}\right) z+\delta\right]\right\},
\end{aligned}
$$

where $\delta=\delta_{1}-\delta_{2}$ is the difference of the initial phases of states with baryon spin polarization along and opposite to the $z$ axis $\left(c_{1}=\left|c_{1}\right| e^{i \delta_{1}} ; c_{2}=\left|c_{2}\right| e^{i \delta_{2}}\right)$. If at $z=0$, the baryon is polarized along $x$, i.e.,

$$
c_{1}=c_{2}=\frac{1}{\sqrt{2}}, \quad \delta=0,
$$

then with growing $z$ the polarization opposite to $x$ appears and further change of polarization looks like oscillations. When baryons pass through the target, one of the components decays more strongly and the baryon beam eventually becomes polarized along or opposite to the $z$ axis. When a beam polarized along the $z$ axis is incident onto the target, no oscillations emerge: only damping occurs. Using (28), one can find the baryon polarization vector

$$
\vec{p}_{n}=\frac{\langle\psi|\vec{\sigma}| \psi\rangle}{\langle\psi \mid \psi\rangle} .
$$

As a result,

$$
\begin{aligned}
& p_{n x}=2 \operatorname{Re}\left(c_{1}^{*} c_{2} \psi_{+}^{*} \psi_{-}\right)\langle\psi \mid \psi\rangle^{-1} \\
& p_{n y}=2 \operatorname{Im}\left(c_{1}^{*} c_{2} \psi_{+}^{*} \psi_{-}\right)\langle\psi \mid \psi\rangle^{-1}, \\
& p_{n z}=\left(\left|c_{1} \psi_{+}\right|^{2}-\left|c_{2} \psi_{-}\right|^{2}\right)\langle\psi \mid \psi\rangle^{-1} .
\end{aligned}
$$

Suppose that baryon spin in vacuum is directed perpendicular to the polarization vector of a nuclei. Choose this direction as the $x$ axis. In this case

$$
c_{1}=c_{2}=1 / \sqrt{2} .
$$

Using relations (33), we obtain

$$
\begin{aligned}
p_{n x} & =\cos \left[k \operatorname{Re}\left(n_{+}-n_{-}\right) z\right] e^{-k \operatorname{Im}\left(n_{+}+n_{-}\right) z}\langle\psi \mid \psi\rangle^{-1}, \\
p_{n y} & =-\sin \left[k \operatorname{Re}\left(n_{+}-n_{-}\right) z\right] e^{-k \operatorname{Im}\left(n_{+}+n_{-}\right) z}\langle\psi \mid \psi\rangle^{-1}, \\
p_{n z} & =\frac{1}{2}\left(e^{-2 k \operatorname{Im}\left(n_{+}\right) z}-e^{2 k \operatorname{Im}\left(n_{-}\right) z}\right)\langle\psi \mid \psi\rangle^{-1} \\
p_{x}^{2}+p_{y}^{2}+p_{z}^{2}=1 . &
\end{aligned}
$$

According to (34) when a baryon penetrates into the interior of the target, its polarization vector rotates about the particle's momentum direction $\vec{n}$ through the angle

$$
\begin{aligned}
\theta & =k \operatorname{Re}\left(n_{+}-n_{-}\right) z \\
& =\frac{2 \pi \rho}{k} \operatorname{Re}\left(f_{+}-f_{-}\right) z \\
& =\frac{\operatorname{Re}\left(U_{W}^{+}-U_{W}^{-}\right)}{\hbar} \frac{z}{c} .
\end{aligned}
$$

At the same time, when baryons pass through matter, the transverse components $p_{n x}$ and $p_{n y}$ of the polarization vector decay because baryon absorption depends on spin orientation, and finally the beam appears to be polarized along or opposite to the $z$ axis. Thus, the dependence of baryon absorption in the target on the orientation of their spin results in the fact that polarization vector $\vec{p}_{n}$ (recall that $\left|\vec{p}_{n}\right|=1$ ) not only rotates about the $z$ axis (about the direction of momentum), but also turns to the $z$ axis (the end point of the polarization vector moves along the unit sphere). If the dependence of absorption on spin orientation can be neglected, the polarization vector rotates about the direction of particle momentum $\vec{\beta}$ only in the $(x, y)$ plane. In terms of kinematics, this phenomenon is analogous to the light polarization plane rotation in a magnetic field (the Faraday effect), while spin oscillations along and opposite to the direction of the $x$ axis are analogous to the transitions $K^{0} \rightleftarrows \bar{K}^{0}$ occurring in regeneration of neutral $K$-mesons (see e.g., [25]).

Let us now consider a particle moving in the straight (unbent) crystal. The expression for $U$ contains a group of terms proportional to either electric field projection onto the $x$ axis or derivative of electrons and nuclei density $\frac{d N_{e(\text { nuc })}(x)}{d x}$. As a result, a particle moving between the planes 
experiences the influence of pseudomagnetic fields that reverse sign due to transverse oscillations of a channeled particle. This leads to the fact that the total spin rotation in such fields is suppressed (although suppression fades with growth of particle energy). The argument mentioned above does not concern the spin rotation effect and spin dichroism caused by weak P-odd T-even interaction. This effect increases with growth of crystal thickness (the effect also occurs in amorphous medium) [10].

Equations describing spin rotation in a bent crystal can be obtained by the following approach [5,7]. Spin wave function $|\Psi(t)\rangle$ meets the equation as follows:

$$
i h \frac{\partial|\Psi(t)\rangle}{\partial t}=\hat{U}_{\mathrm{eff}}|\Psi(t)\rangle .
$$

Baryon polarization vector $\vec{\xi}$ can be expressed as follows:

$$
\vec{\xi}=\frac{\langle\Psi(t)|\vec{\sigma}| \Psi(t)\rangle}{\langle\Psi(t) \mid \Psi(t)\rangle}
$$

Thus the equation for spin rotation of a particle $(\gamma \gg 1)$, which moves in a bent crystal, reads as follows [7] (also see the Appendix):

$$
\begin{aligned}
\frac{d \vec{\xi}}{d t}= & {\left[\vec{\xi} \times \vec{\Omega}_{m s \mathrm{~s}}\right]-\frac{2}{\hbar}\left[\delta_{m}(x)+\delta_{s 0}(x)\right]\left\{\vec{N}_{m}-\vec{\xi}\left(\vec{N}_{m} \cdot \vec{\xi}\right)\right\} } \\
& +\left[\vec{\xi} \times \vec{\Omega}_{T}\right]+\frac{2}{\hbar}\left[\delta_{\mathrm{EDM}}(x)+\delta_{\mathrm{Te}}(x)+\delta_{T \mathrm{nuc}}(x)\right] \\
& \times\left\{\vec{N}_{T}-\vec{\xi}\left(\vec{N}_{T} \cdot \vec{\xi}\right)\right\}+\left[\vec{\xi} \times \vec{\Omega}_{W}\right]-\frac{2}{\hbar} \delta_{W}\{\vec{\beta}-\vec{\xi}(\vec{\beta} \cdot \vec{\xi})\},
\end{aligned}
$$

where

$$
\begin{aligned}
\vec{\Omega}_{\mathrm{mso}} & =\vec{\Omega}_{\mathrm{MDM}}+\vec{\Omega}_{\mathrm{so}}=-\left(\frac{e(g-2)}{2 m c} E_{x}(x)+\frac{2}{\hbar} \alpha_{\mathrm{so}}(x)\right) \vec{N}_{m}, \\
\vec{\Omega}_{T} & =\vec{\Omega}_{\mathrm{EDM}}+\vec{\Omega}_{\mathrm{Ten}}=\frac{2}{\hbar}\left[d E_{x}(x)+\alpha_{\mathrm{Te}}(x)+\alpha_{T \mathrm{nuc}}(x)\right] \vec{N}_{T}, \\
\vec{\Omega}_{w} & =\frac{2}{\hbar} \alpha_{w} \vec{\beta} .
\end{aligned}
$$

Let us note that vector $[\vec{\beta} \times \vec{E}]$ is parallel to vector $\vec{N}_{m}=$ $\left[\vec{\beta} \times \vec{n}_{x}\right]$ and $\vec{N}_{m}=-\vec{N}$ [see (A17)], $\vec{\beta}=\frac{\vec{k}}{k}$ is the unit vector along the direction of particle momentum, vector $\vec{E}$ is parallel to $\vec{N}_{T}=\vec{n}_{x}$ and $\vec{n}_{x}$ is the unit vector along axis $x$. Let us compare Eq. (38) with (1), which has the form as follows:

$$
\frac{d \vec{\xi}}{d t}=\left[\vec{\xi} \times \vec{\Omega}_{\mathrm{magn}}\right]+\left[\vec{\xi} \times \vec{\Omega}_{\mathrm{EDM}}\right]
$$

According to (38) the baryon spin rotates around three axes [5,6]: the effective magnetic field direction $\vec{N}_{m}||[\vec{\beta} \times \vec{E}]$, the electric field direction $\vec{N}_{T} \| \vec{E}$ and the momentum direction $\vec{\beta}$. Nonelastic processes in crystals result in a new effect: those terms in (38), which include deltas $\left(\delta_{m}, \delta_{s 0}, \delta_{\mathrm{EDM}}\right.$, etc.), lead to the appearance of a polarization vector component directed along vectors $\vec{N}_{m}$, $\vec{N}_{T}$ and $\vec{\beta}$. Therefore, spin dichroism induces polarization to particles moving in a bent crystal. Being measured this polarization gives information about MDM/EDM and other interactions. As it is mentioned before, the use of initially nonpolarized beams and polarized crystals could enhance sensitivity of measurements, when polarization of the transmitted through the crystal beam is analyzed. Let us pay attention to the fact that appearance of the spin component directed along the effective magnetic field $B^{*}$ $\left(\vec{N}_{m}\right.$ direction) is caused by both T-odd spin rotation around the direction of the electric field $\vec{E}\left(\vec{N}_{T}\right.$ direction) and spin dichroism due to nonelastic processes at the interaction of the particle magnetic moment with atoms in the bent crystal. It can be seen that appearance of such a spin component imitates the result of the T-noninvariant rotation (Figs. 1 and 2).

Contributions to Eq. (38), which are caused by the interaction between baryon and nuclei, depend on distribution of nuclei density $N_{\text {nuc }}(x)$ [see terms proportional to $\left.\alpha_{s 0}(x), \delta_{s 0}(x), \alpha_{T \text { nuc }}(x), \delta_{T \text { nuc }}(x)\right]$. As a result, for positively charged particles, moving in the channel along the trajectories located in the center of the channel, such contributions are suppressed. Thus, according to (38), when one conducts and interprets experiments aimed for measuring the EDM, one should consider the fact that the angle of spin rotation provides information about the sum of contributions to T-noninvariant rotation. The mentioned rotation is determined by both the EDM and the short-range $C P$-noninvariant interactions. Nonelastic T-noninvariant

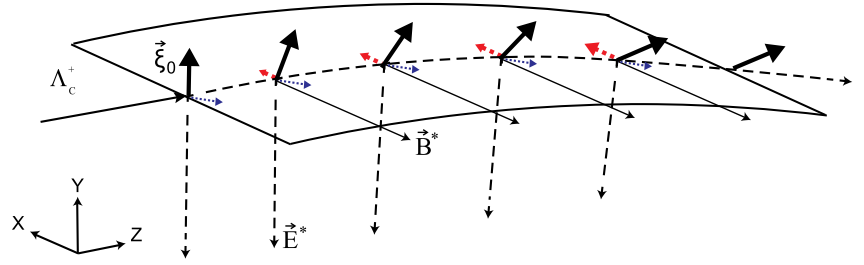

FIG. 1. Spin rotation caused by magnetic moment and T-reversal violation interactions (including EDM). Black arrows represent spin rotation about the effective magnetic field (about bent axis, direction $\vec{N}_{m}$ ), red arrows represent spin component caused by EDM (direction $\vec{N}_{T}$ ), purple arrows represent the new effect-appearance of the spin component directed along $\vec{N}_{m}$ owing to the spin dichroism (spin rotation and dichroism in direction $\vec{N}_{T}$ owing to T-reversal violation and P-violating interactions are not shown here for simplicity). 


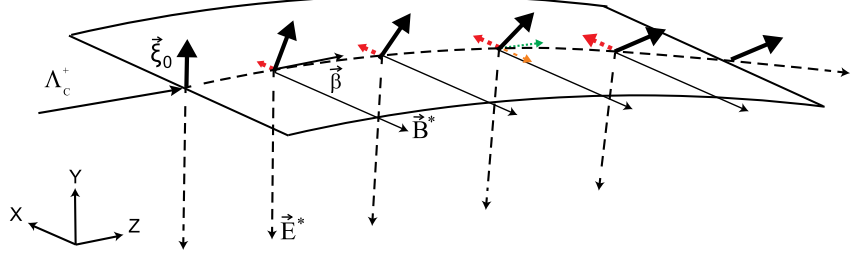

FIG. 2. Spin rotation caused by magnetic moment, T-reversal violation interactions (including EDM), P-violation spin rotation about direction $\vec{\beta}$ (orange arrow) and spin component in direction $\vec{\beta}$ caused by spin dichroism (green arrow). Spin components caused by spin dichroism in direction $\vec{N}_{m}$ and direction $\vec{N}_{T}$ are not shown for simplicity.

processes also lead to spin dichroism in the direction of $\vec{N}_{T}$ that gives additional opportunities for EDM measurement. Let us consider the contribution to the angle of spin rotation caused by spin dichroism in direction $\vec{N}_{m}$. According to [5-7] coefficient $\delta_{m}$ reads as follows:

$$
\begin{aligned}
\delta_{m}= & \frac{1}{4 d_{y} d_{z} m c^{2}}\left(\frac{g-2}{2}\right) \frac{\partial}{\partial x} \overline{\delta V^{2}(x)} \\
= & \frac{1}{4 d_{y} d_{z}} m c^{2}\left(\frac{g-2}{2}\right) \frac{\partial}{\partial x} \int\left\{\overline{\left[\int V_{\text {coul }}(x, y, z) d z\right]^{2}}\right. \\
& \left.-\left[\int \overline{V_{\text {coul }}(x, y, z)} d z\right]^{2}\right\} d y
\end{aligned}
$$

where $\quad V_{\text {coul }}(x, y, z)=\sum_{i} V_{e}\left(x-x_{i}, y-y_{i}, z-z_{i}\right)-$ $V_{\text {nuc }}\left(x-\eta_{f x}, y-\eta_{f y}, z-\eta_{f z}\right), x_{i}, y_{i}, z_{i}$ are the coordinates of the $i$ th electron in the atom, $\eta_{f x}, \eta_{f y}, \eta_{f z}$ are the coordinates of the atom nucleus. Let us choose the position of equilibrium point for the oscillating nucleus as the origin of coordinates. The overline denotes averaging of positions of electrons and nucleus over the distribution of electrons and nucleus positions in the atom; in other words, it denotes averaging with the wave functions of atoms in the crystal.

To conduct estimations the shielded Coulomb potential is used for Coulomb energy of interactions between baryon and electrons; electrons are supposed to be distributed uniformly in the area, which dimensions are about the shielding radius. In this case the following estimations for $\delta_{m}$ can be obtained: $\delta_{m} \sim 10^{8}-10^{9} \mathrm{~s}^{-1}$ depending on the position of the baryon trajectory in the planar channel. According to $[1,2]$ the expected experimental sensitivity for EDM is $e d \sim 10^{-17} e \mathrm{~cm}$. Spin rotation frequency reads as follows: $\Omega_{\mathrm{EDM}}=\frac{2 e d E}{\hbar}$. The magnitude of the $E$-field, which affects baryons in a bent crystal, can be obtained from expression $E=\frac{m \gamma c^{2}}{e R}$, where $R$ is the radius of crystal curvature. Therefore $\Omega_{\mathrm{EDM}}=2 \frac{d}{R} \frac{W}{\hbar}$, where $W$ is the energy of baryon. For $R=30 \mathrm{~m}, d \sim 10^{-17} \mathrm{~cm}$ and $W=1 \mathrm{TeV}$ we have $\Omega_{\mathrm{EDM}} \simeq 10^{7} \mathrm{~s}^{-1}$. As a result, the nonelastic processes, which are caused by magnetic moment scattering, can imitate the EDM contribution. Surely, a more detailed computer simulation is needed. The contributions of P-odd and T-even rotation effect to the general spin rotation can be evaluated in the following way. Precession frequency $\Omega_{w}$ is determined by the real part of the amplitude of baryon weak scattering by an electron (nucleus). This amplitude can be evaluated by Fermi theory $[25,26]$ for the energies, which are necessary for $\mathrm{W}$ and $\mathrm{Z}$ boson production or smaller:

$\operatorname{Re} B \sim G_{F} k=10^{-5} \frac{1}{m_{p}^{2}} k=10^{-5} \frac{\hbar}{m_{p} c} \frac{m \gamma}{m_{p}}=10^{-5} \lambda_{c p} \frac{m \gamma}{m_{p}}$,

where $G_{F}$ is the Fermi constant, $m_{p}$ is the proton mass, $\lambda_{c p}$ is the proton Compton wavelength. For particles with energy from hundreds of $\mathrm{GeV}$ to $\mathrm{TeV} \operatorname{Re} B \sim G_{F} k=$ $10^{-16} \mathrm{~cm}$. For different particle trajectories in a bent crystal the value of precession frequency $\Omega_{w}$ could vary in the range $\Omega_{w} \simeq 10^{3}-10^{4} \mathrm{~s}^{-1}$. Therefore, when a particle passes $10 \mathrm{~cm}$ in a crystal, its spin undergoes additional rotation around the momentum direction at angle $\vartheta_{p} \simeq 10^{-6}-10^{-7} \mathrm{rad}$. Such angle of rotation is small as compared to the one caused by EDM $d \backsim 10^{-17} \mathrm{~cm}$ (at such EDM value precession frequency $\Omega_{\mathrm{EDM}} \backsim 10^{7} \mathrm{~s}^{-1}$, which leads to the rotation angle $\vartheta \backsim 10^{-3}-10^{-4} \mathrm{rad}$ ). However, the effect might be greater for heavy baryons. The mechanism causing the effect growth for heavy baryons is similar to that for their EDM growth (see the explanation for the growth of constant $d_{T}$ in [7]). Absorption caused by the parity violating weak interaction also contributes to change in the spin direction [see the terms proportional to $\delta_{W}$ in (38)]. This change in spin direction is described by the imaginary part of weak scattering amplitude and is proportional to the difference of total scattering cross sections $\sigma_{\uparrow \uparrow}-\sigma_{\downarrow \uparrow}[5,6]$. This difference is proportional to the factor, which is determined by interference of Coulomb and weak interactions for baryon ( $\tau$-lepton) scattering by an electron, as well as by interference of strong (Coulomb) and weak interactions for particle scattering by nuclei $[5,6]$ :

$$
\begin{gathered}
\sigma_{\uparrow \uparrow(\downarrow \uparrow)}=\int\left|f_{c(\mathrm{nuc})}+B_{0 w} \pm B_{w}\right|^{2} d \Omega, \\
\sigma_{\uparrow \uparrow}-\sigma_{\downarrow \uparrow}=2 \int\left[\left(f_{c(\text { nuc })}+B_{0 w}\right) B^{*}+\left(f_{c(\text { nuc })}+B_{0 w}\right)^{*} B\right] d \Omega .
\end{gathered}
$$

When baryon trajectory passes in the area, where collisions with nuclei are important (this occurs in the vicinity of potential barrier for positively charged particles), the value 


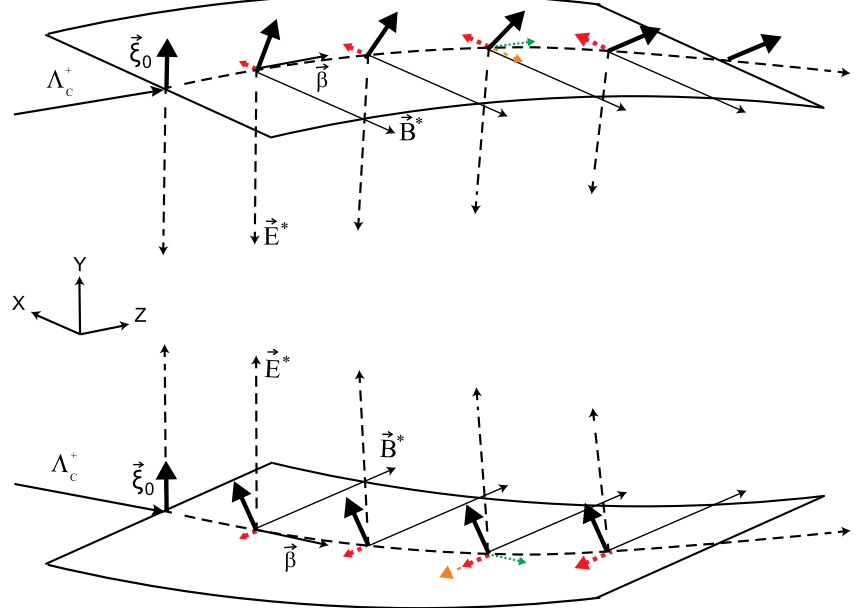

FIG. 3. By turning the crystal $180^{\circ}$ around the direction of incident baryon momentum one could observe that P-odd spin rotation does not change, while the sign of MDM and T-odd spin rotations does due to change of the electric field direction. Subtracting results of measurements for two opposite crystal positions one could obtain the angle of rotation, which does not depend on P-odd effect.
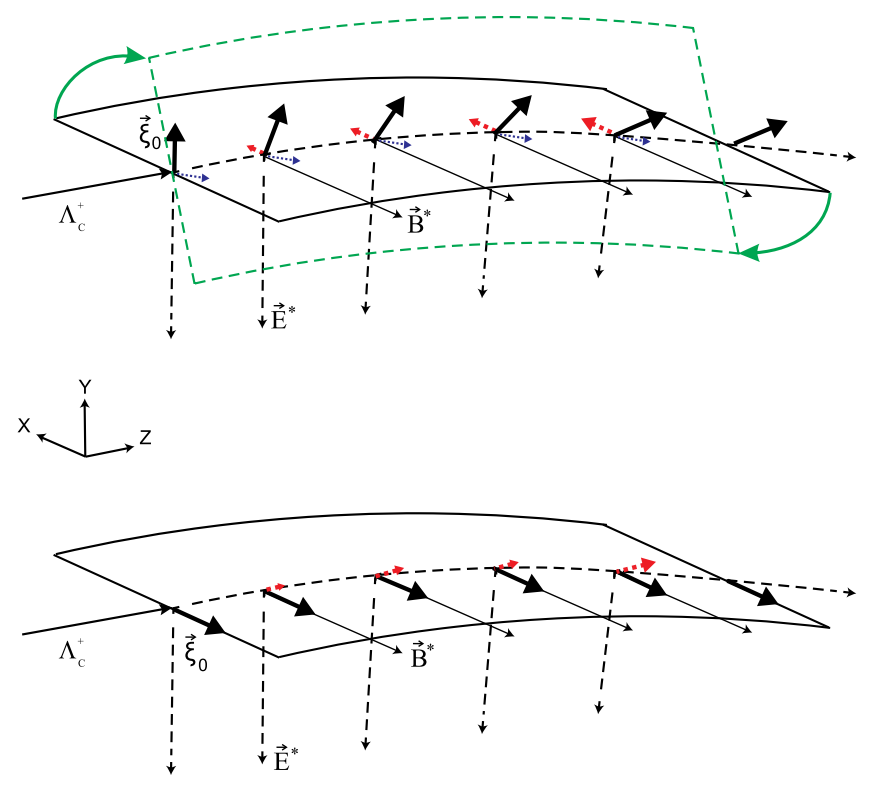

FIG. 4. Separation of the contributions caused by MDM and T-odd spin rotation is possible when comparing experimental results for two initial orientations of polarization vector $\vec{\xi}_{0}$. Namely: $\vec{\xi}_{0} \| \vec{N}_{m}$ and $\vec{\xi}_{0} \| \vec{N}_{T}$, i.e., the initial $\vec{\xi}_{0}$ is parallel to the bending axis of the crystal or $\vec{E}^{*}$. In a real situation rotating the crystal by $90^{\circ}$ so that the direction of $\vec{\xi}_{0}$ is parallel to $\vec{B}^{*}$ can be more convenient. Let us remind that the polarization of the $\Lambda_{c}^{+}$is perpendicular to the production plane. Purple arrows represent the new effect-appearance of the spin component directed along $\vec{N}_{m}$ owing to the spin dichroism caused by baryon ( $\tau$-lepton) scattering due to magnetic moment interaction with Coulomb field of electrons and nuclei.
$\delta_{W} \sim 10^{6}-10^{7} \mathrm{~s}^{-1}$. Similar to real part $\operatorname{Re} B$ for the case of heavy baryons the difference in cross sections grows. Multiple scattering also contributes to spin rotation and depolarization $[5,10,11]$. Particularly, due to interference of magnetic, weak and coulomb interactions, the root-meansquare scattering angle appears changed and dependent on spin orientation with respect to vectors $\vec{N}_{m}, \vec{N}_{T}$ and $\vec{\beta}$. When measuring MDM and T-odd spin rotation in a bent crystal, one can eliminate parity violating rotation in the following way (see Fig. 3).

By turning the crystal $180^{\circ}$ around the direction of incident baryon momentum one could observe that P-odd spin rotation does not change, while the sign of MDM and T-odd spin rotations does due to change of the electric field direction. Subtracting results of measurements for two opposite crystal positions one could obtain the angle of rotation, which does not depend on P-odd effect. Such measurement can be made using the idea presented in [2], according to which to control the systematic uncertainties two crystals (with up and down bending) should be used enabling to induce opposite spin precession for channeled baryons. According to analysis [3] such measurements allow to suppress P-odd effect contribution. Separation of two contributions, that caused by MDM and another one caused by T-odd spin rotation, is possible when comparing the experimental results for two initial orientations of polarization vector $\vec{\xi}_{0}$. Namely, $\vec{\xi}_{0} \| \vec{N}_{m}$ and $\vec{\xi}_{0} \| \vec{N}_{T}$, i.e., the initial polarization $\vec{\xi}_{0}$ is directed either along the bending axis of the crystal or along $\vec{E}^{*}$ (see Fig. 4).

\section{CONCLUSION}

The channeled particle, which moves in a crystal, besides electromagnetic interaction experiences weak interaction with electrons and nuclei, as well as strong interaction with nuclei. Measurements of polarization vector and angular distribution of charged and neutral particles scattered by axes (planes) of unbent crystal enable to obtain limits for the EDM value and for the values of constants describing $\mathrm{P}$ - and T-odd interactions beyond the standard model. When analyzing particle spin rotation, which is caused by electric dipole moment interaction with the electric field, one should consider noninvariant spin rotations both P-odd, T-even and P-odd, T-odd, resulting from weak interaction of baryons and $\tau$-leptons with electrons and nuclei. As demonstrated above, spin precession of channeled particles in bent crystals at the LHC gives a unique possibility for measurement of constants determining T-odd, P-odd $(C P)$ violating interactions and $\mathrm{P}$-odd, T-even interactions of baryons and $\tau$-leptons with electrons and nucleus (nucleons) beyond the standard model. As far as proton beams at LHC are nonpolarized, in [1-4] was proposed the experimental approach, which enables obtaining 
polarized beams of short-lived baryons ( $\tau$-leptons) by means of their generation at reactions of a proton with a nucleus. Analysis of baryon ( $\tau$-lepton) polarization is carried by investigation of angular distribution of its decay products. Another approach enabling to produce polarized short-lived baryons and analyze their polarization implies use of a crystal with polarized nuclei [10]. The main systematic effects for the EDM measurements are as follows: rotation of particle magnetic moment, spin dichroism caused by scattering of a baryon ( $\tau$-lepton), which occurs when particle magnetic moment interacts with Coulomb field of electrons and nuclei, and spin dichroism caused by scattering, which occurs due to Podd T-even weak interactions of the particle with electrons and nuclei.

According to [2] the systematic effect caused by rotation of particle magnetic moment can be eliminated by use of two crystals. In this case the sensitivity of EDM $10^{-17} e \mathrm{~cm}$ can be achieved.

According to the results discussed before, using two crystals or rotating one crystal $180^{\circ}$ around the direction of incident baryon momentum one could exclude influence from both $\mathrm{P}$-odd $\mathrm{T}$-even rotation and $\mathrm{P}$-odd $\mathrm{T}$-even spin dichroism on sensitivity of EDM measurements and provide the sensitivity of $\mathrm{EDM} \sim 10^{-17} e \mathrm{~cm}$.

To separate the contributions caused by EDM (T-odd interactions) and magnetic spin dichroism, one can compare experimental results for two different initial orientations of polarization vector $\vec{\xi}_{0}$, namely: $\vec{\xi}_{0} \| \vec{N}_{m}$ and $\vec{\xi}_{0} \| \vec{N}_{T}$, i.e., the initial $\vec{\xi}_{0}$ is parallel to the bending axis of the crystal or the vector $\vec{E}$. In a real situation rotating the crystal by $90^{\circ}$ so that the direction of $\vec{\xi}_{0}$ is parallel to $B^{*}$ can be more convenient. We remind that the spin component caused by magnetic spin dichroism is directed along $\vec{N}_{m} \| \vec{B}^{*}$. This spin component is orthogonal to $\vec{E}^{*}$, around which spin rotation caused by EDM occurs (see Fig. 4). Therefore, the contribution caused by magnetic spin dichroism (it is directed along $\vec{N}_{m} \perp \vec{k}$ ) is suppressed when one measures spin component, which appears due to EDM and is directed along particle momentum (see the lower plot in Fig. 4). As a result, the sensitivity of EDM $\sim 10^{-17} e \mathrm{~cm}$ remains available. To separate P-noninvariant rotation from the MDM- and EDM-induced (T-odd) spin rotations both the method of turning the crystal by $180^{\circ}$ and the method of using two crystals suggested in [2] can be used. To separate contributions caused by MDM and T-odd interactions, two bent crystals placed perpendicular to each other or rotation of the crystal by $90^{\circ}$ can be used.

\section{ACKNOWLEDGMENTS}

The author would like to thank Dr. Nicola Neri for fruitful discussions.

\section{APPENDIX: EFFECTIVE POTENTIAL ENERGY FOR DIFFERENT INTERACTION TYPES}

\section{Effective potential energy of a spin particle moving close to the crystal planes (axes)}

As it is stated above, elastic coherent scattering of a particle by an atom is caused by electromagnetic interaction of the particle with the atom electrons and nucleus as well as weak and strong nuclear interaction with electrons and nucleus. The general expression for the amplitude of elastic scattering of a particle with spin $\frac{1}{2}$ by spinless or unpolarized nuclei (2) can be written as

$$
\begin{aligned}
\hat{F}(\vec{q})= & A_{\text {coul }}(\vec{q})+A_{s}(\vec{q})+\left[B_{\text {magn }}(\vec{q})\right. \\
& \left.+B_{S}(\vec{q})\right] \vec{\sigma}[\vec{\beta} \times \vec{q}]+\left[B_{w e}(\vec{q})+B_{w n u c}(\vec{q})\right] \vec{\sigma} \vec{N}_{w} \\
& +\left[B_{\mathrm{EDM}}(\vec{q})+B_{\mathrm{Te}}(\vec{q})+B_{T \text { nuc }}(\vec{q})\right] \vec{\sigma} \vec{q},
\end{aligned}
$$

where $\vec{q}=\vec{k}^{\prime}-\vec{k}, \vec{\beta}=\frac{\vec{k}}{k}, A_{\text {coul }}(\vec{q})$ is the spin-independent part of the amplitude of elastic Coulomb scattering of the particle by the atom; $A_{s}(\vec{q})$ is the spin-independent part of the scattering amplitude, which is caused by strong interaction (the similar contribution caused by weak interaction is negligibly small and hereafter is omitted).

The spin-dependent amplitude, which is proportional to $B_{\text {magn }}(\vec{q})$, is determined by electromagnetic spin-orbit interaction. The term proportional to $B_{s}(\vec{q})$ is responsible for the contribution of the spin-orbit strong interaction to the scattering process of a baryon by a nucleus.

The term proportional to P-odd pseudoscalar $\vec{\sigma} \vec{N}_{w}$ (unit vector $\vec{N}_{w}=\frac{\vec{k}^{\prime}+\vec{k}}{\left|\vec{k}^{\prime}+\vec{k}\right|}$ ) includes two contributions: (a) contribution to the amplitude proportional to $B_{w e}(q)$, which describes elastic scattering caused by the parity violating weak interaction between the baryon and electrons; (b) contribution to the amplitude proportional to $B_{\text {wnuc }}(\vec{q})$, which describes elastic scattering caused by the parity violating weak interaction between the baryon and the nucleus.

The term proportional to T-odd ( $C P$-odd) pseudoscalar $\vec{\sigma} \vec{q}$ includes three contributions: (a) contribution proportional to $B_{\mathrm{EDM}}(q)$ describes elastic scattering of a baryon, which possesses EDM, by the atom's Coulomb field; (b) contribution proportional to $B_{\mathrm{Te}}(q)$ describes shortrange T-noninvariant interaction between the baryon and electrons; (c) contribution to the amplitude, which is proportional to $B_{T \text { nuc }}(q)$, describes scattering caused by T-noninvariant interaction between the baryon and nucleons.

By using amplitude $\hat{F}(q)$ potential energy $\hat{U}(\vec{r})$ can be expressed as the sum of terms each of them describing contribution of different interactions to $\hat{U}(\vec{r})$ :

$$
\begin{aligned}
\hat{U}(\vec{r})= & U_{\text {coul }}(\vec{r})+U_{S}(\vec{r})+\hat{U}_{\text {magn }}(\vec{r})+\hat{U}_{s p}(\vec{r}) \\
& +\hat{U}_{W}(\vec{r})+\hat{U}_{T}(\vec{r}),
\end{aligned}
$$


where $U_{\text {coul }}(\vec{r})$ is the Coulomb potential energy of interaction between the baryon and the crystal, which is investigated in detail for particles channeling in the crystal; $U_{S}(\vec{r})$ describes the spin-independent contribution of nuclear interactions to the potential energy of interaction with the crystal; $\hat{U}_{\text {magn }}(\vec{r})$ describes the contribution to $\hat{U}(\vec{r})$ caused by interaction between the baryon's magnetic moment and the atom's electric field; $\hat{U}_{\mathrm{sp}}(\vec{r})$ is the contribution caused by spin-orbital nuclear interactions; $\hat{U}_{W}(\vec{r})$ describes the contribution caused by parity violating weak interactions; $\hat{U}_{T}(\vec{r})$ describes the contribution caused by T-violation interactions between the particle and the crystal.

Let us now consider how different terms, which amplitude (A1) includes, contribute to the effective potential energy of particle interaction with the crystal.

The Coulomb amplitude, described by the first term in (A1), leads to a conventional expression for potential energy of interaction between a charged particle and a plane (axis).

The second term $A_{s}(\vec{q})$ is caused by the short-range interaction. Amplitude $A_{s}(\vec{q})$ can be written as

$$
A_{s}(q)=A_{\mathrm{nuc}}(q) \Phi_{\mathrm{osc}}(\vec{q}),
$$

where $A_{\text {nuc }}(q)$ is the spin-independent part of the amplitude of elastic scattering by the resting nucleus, $\Phi_{\text {osc }}(\vec{q})$ is the form factor caused by nucleus oscillations in the crystal.

Owing to the short-range kind of strong interactions amplitude, $A_{\text {nuc }}(\vec{q})$ is equal to zero-angle scattering amplitude $A(0)$ within the range of scattering angles $\vartheta \leq \frac{1}{k R_{\text {osc }}} \ll 1$.

Form-factor $\Phi_{\text {osc }}(\vec{q})$ has the form [8]

$$
\begin{aligned}
\Phi_{\mathrm{osc}}(\vec{q}) & =\sum_{n} \rho_{n}\left\langle\varphi_{n}(r)\left|e^{-i \vec{q} \vec{r}}\right| \varphi_{n}(r)\right\rangle \\
& =\int e^{-i \vec{q} \vec{r}} N_{\mathrm{nuc}}(\vec{r}) d^{3} r,
\end{aligned}
$$

where $\varphi_{n}(r)$ is the wave function describing the vibrational state of nuclei in the crystal, summation $\sum_{n} \rho_{n}$ means statistical averaging with Gibbs distribution over the vibrational states of nucleus in the crystal. Let us remind that squared form-factor $\Phi_{\text {osc }}(\vec{q})$ is equal to Debye-Waller factor, $N_{\text {nuc }}(\vec{r})$ is the probability density for vibrating nuclei to be detected in point $\vec{r}, \int N_{\text {nuc }}(\vec{r}) d^{3} r=1$.

\section{Effective potential energy determined by the anomalous magnetic moment}

According to (A1) the scattering amplitude, which is determined by baryon's anomalous magnetic moment, has the form

$$
\hat{F}_{\text {magn }}(q)=B_{\text {magn }}(q) \vec{\sigma}[\vec{\beta} \times \vec{q}] .
$$

For the first step the perturbation theory can be used and amplitude $\hat{F}^{(1)}$ reads as

$$
\hat{F}_{\text {magn }}^{(1)}(\vec{q})=i f_{\text {coul }}(\vec{q}) \frac{\hbar}{m c}\left(\frac{g-2}{2}\right) \frac{1}{2} \vec{\sigma}[\vec{\beta} \times \vec{q}],
$$

where $f_{\text {coul }}(\vec{q})$ is the amplitude of Coulomb scattering of a baryon by an atom in the first Born approximation; $\vec{\beta}=\frac{\vec{k}}{k}$, $m$ is the baryon mass.

It should be noted that the coefficient in (A6), by which $\vec{\sigma}$ is multiplied, is purely imaginary. Using (A6) one can obtain the expression for effective interaction energy as follows:

$$
\hat{U}_{\text {magn }}(x)=-\frac{e \hbar}{2 m c} \frac{g-2}{2} \vec{\sigma}\left[\vec{E}_{\text {plane }}(x) \times \vec{\beta}\right],
$$

where $\vec{E}_{\text {plane }}(x)$ denotes the electric field, produced in point $x$ by the crystallographic plane. In the axis case $U_{\text {magn }}(\vec{\rho})$ can be obtained by replacement in (A7) of $x$ by $\vec{\rho}$ and of $\vec{E}_{\text {plane }}(x)$ by $\vec{E}_{\text {axis }}(\vec{\rho})$, respectively.

Effective interaction energy (A7) can be rewritten as follows:

$$
\hat{U}_{\text {magn }}=-\frac{e \hbar}{2 m c} \frac{g-2}{2} E_{\text {xplane }}(x) \vec{\sigma} \vec{N},
$$

where $\vec{N}=\left[\vec{n}_{x} \times \vec{\beta}\right]$ is the unit vector, $\vec{n}_{x} \perp \vec{\beta}$, unit vector $\vec{\beta}$ is parallel to the crystallographic plane.

Expression (A8) for the effective potential energy comprises the factor, which is purely real. However, the coefficient in the expression for scattering amplitude $\hat{F}(\vec{q})$, by which $\vec{\sigma}$ is multiplied, has nonzero both real and imaginary parts. Due to this fact, the effective potential energy $\hat{U}$ also has nonzero both real and imaginary parts.

In the second order of perturbation theory this coefficient in amplitude $\hat{F}(\vec{q})$ is not purely imaginary as well-it has a nonzero real part. According to [7] the following expression for the contribution $\tilde{F}^{(2)}(q)$ to the amplitude $\hat{F}(\vec{q})$ can be obtained:

$$
\begin{aligned}
& \tilde{F}^{(2)}(\vec{q}=\vec{\tau})=i \frac{k}{4 \pi \hbar^{2} c^{2}} \iint e^{-i \vec{\tau} \vec{r}_{\perp}}\left\{\overline{\left[\int \hat{V}\left(\vec{r}_{\perp}, z\right) d z\right]^{2}}\right. \\
& \left.-\left[\overline{\int \hat{V}\left(\vec{r}_{\perp}, z\right) d z}\right]^{2}\right\} d^{2} r_{\perp} \text {, }
\end{aligned}
$$

where $\hat{V}\left(\vec{r}_{\perp}, z\right)=\hat{V}_{\text {coul }}\left(\vec{r}_{\perp}, z\right)+\hat{V}_{\text {magn }}\left(\vec{r}_{\perp}, z\right), \hat{V}_{\text {magn }}\left(\vec{r}_{\perp}, z\right)=$ $-\mu_{a} \vec{\sigma}\left[\vec{E}\left(\vec{r}_{\perp}, z\right) \times \vec{\beta}\right], z$ axis of the coordinate system is directed along the unit vector $\vec{\beta}, \vec{\beta}$ is the unit vector directed along the particle momentum before scattering 
$\hbar \vec{k}, \mu_{a}$ is the anomalous magnetic moment of the particle $\mu_{a}=\frac{e \hbar}{2 m c}\left(\frac{g-2}{2}\right)$.

When deriving (A9), the particle energy is considered to be much greater than the electrons' binding energy in atoms and the atoms' binding energy in the crystal. As a result it is possible at first to examine scattering of electrons by a nucleus, which rests in point $r_{i}$, and, then, to average the result over the electron and nuclei positions with wave functions $\left|\Phi_{a}\right\rangle$ (impulse approximation, for example see [31]). The overline in (A9) and hereafter denotes such a kind of averaging. The contribution caused by interference between magnetic and nuclear scattering, and the contributions determined by the particle squared magnetic moment, should complete the expression mentioned above. For positively charged particles, moving far from the top of the potential barrier, the contribution caused by interactions with nuclei is suppressed and is omitted in consideration hereafter. Contributions proportional to the particle squared magnetic moment are smaller than those caused by interference between magnetic and Coulomb scattering and, thus, is also omitted. According to [7] the following expression for the contribution to the effective potential energy caused by the amplitude $\tilde{F}_{\text {magn }}(\vec{\tau})$ can be obtained:

$\hat{U}_{\text {magn }}^{(2)}(x)=-i \frac{1}{4 d_{y} d_{z} m c^{2}}\left(\frac{g-2}{2}\right) \frac{\partial}{\partial x} \overline{\delta V^{2}(x)} \vec{\sigma} \vec{N}$,

where $\vec{N}=\left[\vec{n}_{x} \times \vec{\beta}\right], \vec{n}_{x} \perp \vec{\beta}, \vec{n}_{x}$ is the unit vector along axis $x$,

$$
\begin{aligned}
\overline{\delta V^{2}(x)}= & \int\left\{\overline{\left[\int V_{\text {coul }}(x, y, z) d z\right]^{2}}\right. \\
& -\left[\overline{\left.\left.\int V_{\text {coul }}(x, y, z) d z\right]^{2}\right\} d y .}\right.
\end{aligned}
$$

Similarly for the case of axial channeling (A10) converts to

$\hat{U}_{\text {magn }}^{(2)}(\vec{\rho})=-i \frac{1}{d_{z} m c^{2}}\left(\frac{g-2}{2}\right) \vec{\sigma}\left[\nabla_{\rho} \overline{\delta V^{2}(\vec{\rho})} \times \vec{\beta}\right]$.

In the planar channeling case $\hat{U}_{\text {magn }}$ is determined by

$$
\begin{aligned}
\hat{U}_{\text {magn }}(x)= & -\frac{e \hbar}{2 m c} \frac{g-2}{2} \vec{\sigma}\left[\vec{E}_{\text {plane }} \times \vec{\beta}\right] \\
& -i \frac{1}{4 d_{y} d_{z} m c^{2}}\left(\frac{g-2}{2}\right) \frac{\partial}{\partial x} \overline{\delta V^{2}(x)} \vec{\sigma} \vec{N} \\
= & -\left(\alpha_{m}+i \delta_{m}\right) \vec{\sigma} \vec{N},
\end{aligned}
$$

where

$$
\begin{aligned}
\vec{N} & =\left[\overrightarrow{n_{x}} \times \vec{\beta}\right], \\
\alpha_{m} & =\frac{e \hbar}{2 m c} \frac{g-2}{2} E_{x}, \\
\delta_{m} & =\frac{1}{4 d_{y} d_{z} m c^{2}}\left(\frac{g-2}{2}\right) \frac{\partial}{\partial x} \overline{\delta V^{2}(x)}
\end{aligned}
$$

and $\overline{\delta V^{2}(x)}=\int\left\{\overline{\left[\int V_{\text {coul }}(x, y, z) d z\right]^{2}}-\left[\int V_{\text {coul }}(x, y, z) d z\right]^{2}\right\} d y$ is the mean-square fluctuation of energy of Coulomb interaction between the baryon and the atom.

Similarly for the axial channeling case the following expression can be obtained:

$\hat{U}_{\text {magn }}(\vec{\rho})=-\frac{e \hbar}{2 m c} \frac{g-2}{2} \vec{\sigma}\left[\vec{E}_{\text {axis }} \times \vec{\beta}\right]+\hat{U}_{\text {magn }}^{(2)}(\vec{\rho})$,

where $\hat{U}_{\text {magn }}^{(2)}(\vec{\rho})$ is determined by (A11).

\section{Effective potential energy $\hat{U}$ determined by spin-orbit interaction}

According to (A1) the part of the scattering amplitude caused by strong spin-orbit interaction has the form

$$
\hat{F}_{s p}(\vec{q}=\vec{\tau})=B_{s}(\vec{\tau}) \vec{\sigma}[\vec{\beta} \times \vec{\tau}]
$$

The coefficient $B_{s}(\vec{\tau})$ can be expressed similar to (A3) as follows:

$$
B_{s}(\vec{\tau})=B_{\text {nuc }}(\vec{\tau}) \Phi_{\text {osc }}(\vec{\tau}),
$$

where $B_{\text {snuc }}(\vec{\tau})$ describes scattering by a resting nucleus, $\Phi_{\text {osc }}(\vec{\tau})$ is the form factor determined by nucleus oscillations in a crystal.

In the considered case the short-range character of the nuclear forces and small (as compared to the amplitude of nucleus oscillations) nucleus radius enables assumption $B_{\text {snuc }}(\vec{\tau}) \approx B_{\text {snuc }}(0)$. It is important that the coefficient $B_{s \text { nuc }}(0)$ has nonzero both real and imaginary parts:

$$
B_{\text {snuc }}(0)=B_{\text {snuc }}^{\prime}+i B_{\text {snuc }}^{\prime \prime} \text {. }
$$

This is similar to the amplitude, which describes scattering of the magnetic moment by the atom (nucleus). To obtain the expression for the effective potential energy the summation over $\tau_{x}$ should be conducted in (A14). The resulting expression is similar to that for $\hat{U}_{\text {magn }}$. For example, for planar channeling effective potential energy $\hat{U}$ determined by spin-orbit interaction between baryon and nucleus reads as follows:

$$
\hat{U}_{\mathrm{so}}=-\left(\alpha_{s}+i \delta_{s}\right) \vec{\sigma} \vec{N},
$$

where 


$$
\begin{aligned}
\vec{N} & =\left[\overrightarrow{n_{x}} \times \vec{\beta}\right], \\
\alpha_{s} & =-\frac{2 \pi \hbar^{2}}{m \gamma d_{y} d_{z}} \frac{\partial N_{\mathrm{nuc}}}{\partial x} B^{\prime \prime}, \\
\delta_{s} & =\frac{2 \pi \hbar^{2}}{m \gamma d_{y} d_{z}} B^{\prime} \frac{\partial N_{\mathrm{nuc}}}{\partial x} .
\end{aligned}
$$

In the case of axial channeling,

$$
\hat{U}_{\mathrm{so}}(\rho)=\frac{2 \pi \hbar^{2}}{m \gamma d_{z}}\left(B^{\prime \prime}-i B^{\prime}\right) \vec{\sigma}\left[\vec{\nabla}_{\rho} N_{\text {nuc }}(\vec{\rho}) \times \vec{\beta}\right] .
$$

\section{Effective potential energy $\hat{U}$ determined by P-odd and T-even interactions}

The next group of terms, which are proportional to $B_{w}$, is determined by weak P-odd and T-even interactions. According to (A1) the corresponding terms in the scattering amplitude can be written as

$$
\hat{F}_{w}(\vec{q})=\left[B_{w e}(\vec{q})+B_{w n u c}(\vec{q})\right] \vec{\sigma} \vec{N}_{w} .
$$

Contribution $B_{\text {we }}(\vec{q})$ caused by parity violating weak interaction between the baryon and electrons can be expressed as follows:

$$
B_{w e}(\vec{q})=\tilde{B}_{w e}(\vec{q}) \Phi_{e}(\vec{q}),
$$

where $\tilde{B}_{w e}$ is the coefficient defining baryon elastic scattering amplitude by resting electron $\hat{f}_{w e}(q)=\tilde{B}_{w e} \vec{\sigma} \vec{N}_{w}$, $\Phi_{e}(\vec{q})=\int e^{-i \vec{q} \vec{r}} N_{e}(\vec{r}) d^{3} r, \quad \int N_{e}(\vec{r}) d^{3} r=Z, \quad Z \quad$ is the nucleus charge. Minor corrections caused by thermal oscillations of atoms' centers of gravity are not considered below. To take them into consideration one should multiply $\Phi_{e}(\vec{q})$ by $\Phi_{\text {osc }}(\vec{q})$, which is the form factor defined by oscillations of the atom's nucleus.

Term $B_{\text {wnuc }}(\vec{q})$ [see (A20)], which is caused by parity violating weak interaction between a baryon and a nucleus, reads as follows:

$$
B_{w n u c}(\vec{q})=\tilde{B}_{w \mathrm{nuc}}(\vec{q}) \Phi_{\mathrm{osc}}(\vec{q})
$$

where $\tilde{B}_{w n u c}$ is the coefficient defining the amplitude of baryon elastic scattering by the resting nucleus $\hat{f}_{\text {wnuc }}=\tilde{B}_{w \text { nuc }} \vec{\sigma} \vec{N}_{w}$.

In case when angle $\vartheta \simeq \frac{\tau}{k} \ll 1$ the coefficients $\tilde{B}_{w e}(\vec{q})$ and $\tilde{B}_{\text {wnuc }}(\vec{q})$ can be approximated by $\tilde{B}_{w e}(\vec{q}) \simeq \tilde{B}_{w e}(0)$ and $\tilde{B}_{\text {wпис }}(\vec{q}) \simeq \tilde{B}_{\text {wпис }}(0)$, respectively. As a result expressions for the effective potential energy $\hat{U}_{w}$ of P-violating interaction of a baryon with a crystal plane (axis) were obtained. Effective potential energy $\hat{U}$ determined by P-odd and T-even interactions in the case of plane:
$\hat{U}_{w}(x)=\hat{U}_{w e}(x)+\hat{U}_{w n u c}(x)=-\left[\alpha_{w}(x)+i \delta_{w}(x)\right] \vec{\sigma} \vec{\beta}$,

where

$$
\begin{aligned}
& \alpha_{w}(x)=\frac{2 \pi \hbar^{2}}{m \gamma d_{y} d_{z}}\left[\tilde{B}_{w e}^{\prime}(0) N_{e}(x)+\tilde{B}_{w n u c}^{\prime}(0) N_{\mathrm{nuc}}(x)\right], \\
& \delta_{w}(x)=\frac{2 \pi \hbar^{2}}{m \gamma d_{y} d_{z}}\left[\tilde{B}_{w e}^{\prime \prime}(0) N_{e}(x)+\tilde{B}_{w \mathrm{nuc}}^{\prime \prime}(0) N_{\mathrm{nuc}}(x)\right],
\end{aligned}
$$

where signs ' and " denote the real and imaginary parts of the quantity, respectively.

In the case of axial channeling the same reads as follows:

$$
\begin{aligned}
\hat{U}_{w e}(\vec{\rho})= & -\frac{2 \pi \hbar^{2}}{m \gamma d_{z}}\left\{\tilde{B}_{w e}(0) N_{e}(\vec{\rho})\right. \\
& \left.+\tilde{B}_{w \mathrm{nuc}}(0) N_{\mathrm{nuc}}(\vec{\rho})\right\} \vec{\sigma} \vec{N}_{w}, \\
N_{e(\mathrm{nuc})}(\vec{\rho})= & \int N_{e(\mathrm{nuc})}(\vec{\rho}, z) d z .
\end{aligned}
$$

\section{Effective potential energy $\hat{U}$ determined by the electric dipole moment and other T-noninvariant interactions}

Let us consider now the electric dipole moment and other T-noninvariant contributions to the spin rotation. According to (A1) the corresponding terms in the scattering amplitude can be written as

$$
\hat{F}_{T}(q)=\left[B_{\mathrm{EDM}}(q)+B_{\mathrm{Te}}(q)+B_{T \mathrm{nuc}}(q)\right] \vec{\sigma} \vec{q} .
$$

Let us consider the term $\hat{F}_{\mathrm{EDM}}(q)=B_{\mathrm{EDM}}(\vec{q}) \vec{\sigma} \vec{q}$. The coefficient $B_{\mathrm{EDM}}(q)$ has nonzero both real and imaginary parts $B_{\mathrm{EDM}}(q)=B_{\mathrm{EDM}}^{\prime}+i B_{\mathrm{EDM}}^{\prime \prime}$. By the approach used for deriving $\hat{F}_{\text {magn }}(q)$, for $\hat{F}_{\mathrm{EDM}}(\vec{q})$ one can obtain

$$
\begin{aligned}
\hat{F}_{\mathrm{EDM}}(\vec{q})= & -i \frac{m \gamma d}{2 \pi \hbar^{2}} V_{\text {coul }}(\vec{q}) \vec{\sigma} \vec{q}+\frac{k}{4 \pi \hbar^{2} c^{2}} \\
& \times \iint e^{-i \vec{q}_{\perp} \vec{r}_{\perp}}\left\{\overline{\left[\int \hat{V}\left(\vec{r}_{\perp}, z\right) d z\right]^{2}}\right. \\
& \left.-\left[\int \overline{\hat{V}\left(\vec{r}_{\perp}, z\right)} d z\right]^{2}\right\} d^{2} r_{\perp},
\end{aligned}
$$

where $\hat{V}(\vec{r})=V_{\text {coul }}(\vec{r})+V_{\mathrm{EDM}}(\vec{r}), V_{\mathrm{EDM}}=-D \vec{\sigma} \vec{E}$ is the energy of interaction between the electric dipole moment $D=e d$ and the electric field $\vec{E}$. Let us remind that amplitude $\hat{F}_{T}(\vec{q})$ contains terms caused by EDM and those determined by short-range T-noninvariant interactions of a baryon with electrons and nuclei $B_{\mathrm{Te}}(\vec{q})$ and $B_{T \text { nuc }}(q)$. Contributions caused by these terms should 
also be added to the effective potential energy of the interaction between the baryon and nuclei of the crystal $\hat{U}_{T}(x)$ (see [7]):

$$
\hat{U}_{T}(x)=\hat{U}_{\mathrm{EDM}}+\hat{U}_{\mathrm{Te}}+\hat{U}_{T \mathrm{nuc}}=-\left[\alpha_{T}(x)+i \delta_{T}(x)\right] \vec{\sigma}_{T},
$$

where $\alpha_{T}=\alpha_{\mathrm{EDM}}+\alpha_{\mathrm{Te}}+\alpha_{T \text { nuc }}, \delta_{T}=\delta_{\mathrm{EDM}}+\delta_{\mathrm{Te}}+\delta_{T \text { nuc }}$.

Expressions for coefficients $\alpha_{\mathrm{Te}(\mathrm{nuc})}$ and $\delta_{\mathrm{Te}(\mathrm{nuc})}$ can be evaluated in terms of scattering amplitude in the following way. Let us define the form factor determined by electrons distribution in an atom and nucleus oscillations:

$$
B_{\mathrm{Te}}(\vec{q})=\tilde{B}_{\mathrm{Te}}(\vec{q}) \Phi_{e}(\vec{q}), B_{T \mathrm{nuc}}(\vec{q})=\tilde{B}_{T \mathrm{nuc}}(\vec{q}) \Phi_{\mathrm{osc}}(\vec{q}),
$$

where $\Phi_{e}(\vec{q})=\int e^{-i \vec{q} \vec{r}} N_{e}(\vec{r}) d^{3} r, N_{e}(\vec{r})$ is electron distribution density in the atom, $\int N_{e}(\vec{r}) d^{3} r=Z, Z$ is the nucleus charge, $\Phi_{\mathrm{osc}}(\vec{q})$ is determined by (A4), $\tilde{B}_{\mathrm{Te}}$ is the coefficient defining amplitude of baryon scattering by a resting electron $\hat{f}_{\mathrm{Te}}=\tilde{B}_{\mathrm{Te}}(\vec{q}) \vec{\sigma} \vec{q}, \tilde{B}_{\text {nuc }}(q)$ is the coefficient defining amplitude of baryon scattering by a resting nucleus $\hat{f}_{T \text { nuc }}=\tilde{B}_{\text {nuc }}(\vec{q}) \vec{\sigma} \vec{q}$. Let us remind that in compliance with [7] the contribution caused by elastic coherent scattering should be subtracted from amplitude $B_{T}$. However, at high energies this contribution is negligibly small in comparison with nonelastic contributions to the amplitude and, therefore, can be omitted.

Due to the short-range character of T-noninvariant interactions at angle $\vartheta \simeq \frac{\tau}{k} \ll 1$ coefficients $\tilde{B}_{\mathrm{Te}}(\vec{q}) \simeq \tilde{B}_{\mathrm{Te}}(0)$ and $\tilde{B}_{\text {nuc }}(\vec{q}) \simeq \tilde{B}_{\text {nuc }}(0)$.

Due to the short-range character of T-noninvariant interactions at angle $\vartheta \simeq \frac{\tau}{k} \ll 1$ coefficients $\tilde{B}_{\mathrm{Te}}(\vec{q}) \simeq \tilde{B}_{\mathrm{Te}}(0)$ and $\tilde{B}_{\text {nuc }}(\vec{q}) \simeq \tilde{B}_{\text {nuc }}(0)$. As a result, the following expressions can be obtained:

$$
\begin{aligned}
\hat{U}_{\mathrm{Te}}(x) & =i \frac{2 \pi \hbar^{2}}{m \gamma d_{y} d_{z}} \tilde{B}_{\mathrm{Te}}(0) \frac{d N_{e}(x)}{d x} \vec{\sigma} \vec{N}_{T}, \\
\hat{U}_{T \mathrm{nuc}}(x) & =i \frac{2 \pi \hbar^{2}}{m \gamma d_{y} d_{z}} \tilde{B}_{T \text { nuc }}(0) \frac{d N_{\mathrm{nuc}}(x)}{d x} \vec{\sigma} \vec{N}_{T}, \\
N_{e(\text { nuc })}(x) & =\int N_{e(\text { nuc })}(x, y, z) d y d z .
\end{aligned}
$$

Coefficients $\tilde{B}_{\mathrm{Te}}(0)$ and $\tilde{B}_{T \text { nuc }}(0)$ are complex values:

$$
\tilde{B}_{\mathrm{Te}(\mathrm{nuc})}(0)=\tilde{B}_{\mathrm{Te}(\mathrm{nuc})}^{\prime}+i \tilde{B}_{\mathrm{Te}(\mathrm{nuc})}^{\prime \prime} \text {. }
$$

As a result, T-violating interactions lead to a contribution to potential energy (A28). Energy of electric dipole moment interaction with electric field reads as follows:
$\hat{U}_{\mathrm{EDM}}=-e d E_{\mathrm{pl}}(x) \vec{\sigma} \vec{N}_{T}-i \frac{d}{2 d_{y} d_{z} \hbar c} \frac{\partial}{\partial x} \overline{\delta V^{2}(x)} \vec{\sigma}_{T}$,

where unit vector $\vec{N}_{T}$ is orthogonal to the crystal plane, $\vec{E}_{\mathrm{pl}}(x)=E_{x} \vec{N}_{T}$.

Evidently, $\hat{U}_{\mathrm{EDM}}$ can be expressed as

$$
\hat{U}_{\mathrm{EDM}}=-\left(\alpha_{\mathrm{EDM}}+i \delta_{\mathrm{EDM}}\right) \vec{\sigma} \vec{N}_{T} .
$$

Similar to $\hat{U}_{\text {magn }}$ energy $\hat{U}_{\mathrm{EDM}}$ has nonzero both real and imaginary parts. The expression for $\hat{U}_{\text {magn }}$ converts to $\hat{U}_{\text {EDM }}$ by replacement $\frac{g-2}{2} \rightarrow 2 \frac{d}{\lambda_{c}}\left(\lambda_{c}=\frac{\hbar}{m c}\right.$ is the Compton wavelength of the particle) and $\vec{N} \rightarrow \vec{N}_{T}$. Short-range Todd interactions give contributions to the effective potential energy that can be written as

$$
\hat{U}_{\mathrm{Te}(\mathrm{nuc})}(x)=-\left[\alpha_{\mathrm{Te}(\mathrm{nuc})}+i \delta_{\mathrm{Te}(\mathrm{nuc})}\right] \vec{\sigma} \vec{N}_{T},
$$

where

$$
\begin{aligned}
& \alpha_{\mathrm{Te}(\mathrm{nuc})}=\frac{2 \pi \hbar^{2}}{m \gamma d_{y} d_{z}} \tilde{B}_{\mathrm{Te}(\mathrm{nuc})}^{\prime \prime} \frac{d N_{e(\mathrm{nuc})}(x)}{d x}, \\
& \delta_{\mathrm{Te}(\mathrm{nuc})}=\frac{2 \pi \hbar^{2}}{m \gamma d_{y} d_{z}} \tilde{B}_{\mathrm{Te}(\mathrm{nuc})}^{\prime} \frac{d N_{e(\mathrm{nuc})}(x)}{d x} .
\end{aligned}
$$

For interaction with crystal axis,

$\hat{U}_{\mathrm{Te}(\mathrm{nuc})}(\vec{\rho})=-\frac{2 \pi \hbar^{2}}{m \gamma d_{z}}\left(\tilde{B}_{\mathrm{Te}(\mathrm{nuc})}^{\prime \prime}+i \tilde{B}_{\mathrm{Te}(\mathrm{nuc})}^{\prime}\right) \vec{\sigma} \vec{\nabla}_{\rho} N_{\mathrm{Te}(\mathrm{nuc})}(\vec{\rho})$.

Thus in the experiment aimed to obtain the limit for the EDM value, the limits for the scattering amplitudes, which are determined by short-range $\mathrm{T}(C P)$-noninvariant interactions between baryons ( $\tau$-leptons) and electrons, and nuclei, will be obtained as well. The obtained values of these amplitudes for different interaction types allows one to restore the values of corresponding constants, too. It should be noted that constant of interaction between a heavy baryon (tau-lepton) and an electron (nucleus, nucleon) can be greater than that for light particle interaction. This effect can be explained by the reasoning similar to that explaining the expected EDM growth for a heavy baryon [6]. T-odd interaction mixes heavy baryon stationary states with different parity more effectively that for light baryons due to probably smaller spacing between energy levels corresponding to these states. 
[1] E. Bagli, L. Bandiera, G. Cavoto, V. Guidi, L. Henry, D. Marangotto, F. Martinez Vidal, A. Mazzolari, A. Merli, N. Neri, and J. Ruiz Vidal, Electromagnetic dipole moments of charged baryons with bent crystals at the LHC, Eur. Phys J. C 77, 828 (2017).

[2] F. J. Botella, L. M. Garcia Martin, D. Marangotto, F. Martinez Vidal, A. Merli, N. Neri, A. Oyanguren, and J. Ruiz Vidal, On the search for the electric dipole moment of strange and charm baryons at LHC, Eur. Phys J. C 77, 181 (2017).

[3] J. Fu, M. A. Giorgi, L. Henry, D. Marangotto, F. Martinez Vidal, A. Merli, N. Neri, and J. Ruiz Vidal, Novel Method for the Direct Measurement of the $\tau$ Lepton Dipole Moments, Phys. Rev. Lett. 123, 011801 (2019).

[4] A. S. Fomin, A. Yu. Korchin, A. Stocchi, S. Barsuk, and P. Robbe, Feasibility of tau lepton electromagnetic dipole moments measurement using bent crystal at the LHC, J. High Energy Phys. 03 (2019) 156.

[5] V. G. Baryshevsky, On the search for the electric dipole moment of strange and charm baryons at LHC and parity violating $(\mathrm{P})$ and time reversal $(\mathrm{T})$ invariance violating spin rotation and dichroism in crystal, arXiv:1708.09799.

[6] V. G. Baryshevsky, Electromagnetic dipole moments and time reversal violating interactions for high energy charged baryons in bent crystals at LHC, Eur. Phys. J. C 79, 350 (2019); Time reversal invariance violation for high energy charged baryons in bent crystals, arXiv:1803.05770v1.

[7] V. G. Baryshevsky, Electromagnetic dipole moment and time reversal invariance violating interactions for high energy short-lived particles in bent and straight crystals at Large Hadron Collider, arXiv:1812.00428v1.

[8] V. B. Berestetskii, E. M. Lifshitz, and L. P. Pitaevskii, Quantum Electrodynamics, Course of Theoretical Physics, edited by L. D. Landau and E. M. Lifshitz, 2nd ed. (Butterworth-Heinemann, London, 1982), Vol. 4.

[9] V. G. Baryshevsky, Spin rotation of ultrarelativistic particles passing through a crystal, Pis'ma Zh. Tekh. Fiz. 5, 182 (1979).

[10] V. G. Baryshevsky, High-Energy Nuclear Optics of Polarized Particles (World Scientific Publishing Company, Singapore, 2012), p. 640.

[11] V. G. Baryshevsky, Spin rotation and depolarization of high-energy particles in crystals at LHC and FCC energies. The possibility to measure the anomalous magnetic moments of short-lived particles and quadrupole moment of $\Omega$-hyperon, Nucl. Instrum. Methods Phys. Res., Sect. B 402, 5 (2017).

[12] V. G. Baryshevsky, The possibility to measure the magnetic moments of short-lived particles (charm and beauty baryons) at LHC and FCC energies using the phenomenon of spin rotation in crystals, Phys. Lett. B 757, 426 (2016).

[13] V. V. Baublis et al. (E761 Collaboration), First observation of spin precession of polarized $\Sigma^{+}$hyperons channeled in bent crystals, LNPI Research Report, 1990-1991 (1992), pp. 24-26.
[14] D. Chen et al., First Observation of Magnetic Moment Precession of Channeled Particles in Bent Crystals, Phys. Rev. Lett. 69, 3286 (1992).

[15] A. V. Khanzadeev, V. M. Samsonov, R. A. Carrigan, and D. Chen, Experiment to observe the spin precession of channeled relativistic $\Sigma^{+}$hyperons, Nucl. Instrum. Methods Phys. Res., Sect. B 119, 266 (1996).

[16] V. G. Baryshevsky, Spin rotation and depolarization of high-energy particles in crystals at Hadron Collider (LHC) and Future Circular Collider (FCC) energies and the possibility to measure the anomalous magnetic moments of short-lived particles, arXiv:1504.06702.

[17] O. A. Bezshyyko, L. Burmistrov, A. S. Fomin, S. P. Fomin, A. Yu. Korchin, I. V. Kirillin, L. Massacrier, A. Natochii, P. Robbe, W. Scandale, N.F. Shul'ga, and A. Stocchi, Feasibility of measuring the magnetic dipole moments of the charm baryons at the LHC using bent crystals, J. High Energy Phys. 08 (2017) 120.

[18] V. G. Baryshevsky and A. R. Bartkevich, Tensor polarization of deuterons passing through matter, J. Phys. G 39, 125002 (2012).

[19] G. W. Ford and C. W. Hirt, Report, Michigan Univ., 1961 (unpublished).

[20] S. R. Mane, Yu. M. Shatunov, and K. Yokoya, Spinpolarized charged particle beams in high-energy accelerators, Rep. Prog. Phys. 68, 1997 (2005).

[21] V. P. Gudkov, H. Xiao-Gang, and B. H. J. McKeller, CPodd nucleon potential, Phys. Rev. C 47, 2365 (1993).

[22] T. E. Chupp, P. Fierlinger, M. J. Ramsey-Musolf, and J. T. Singh, Electric dipole moments of atoms, molecules, nuclei, and particles, Rev. Mod. Phys. 91, 015001 (2019).

[23] I. B. Khriplovich and S. K. Lamoreaux, CP Violation Without Strangeness: Electric Dipole Moments of Particles, Atoms, and Molecules (Springer, New York, 2011).

[24] V. L. Lyuboshits, The spin rotation at deflection of relativistic charged particle in electric field, Yad. Fiz. 31, 986 (1980) [Sov. J. Nucl. Phys. 31, 509 (1980)].

[25] E. D. Commins and P. H. Bucksbaum, Weak Interactions of Leptons and Quarks (Cambridge University Press, Cambridge, England, 1983).

[26] E. Leader and E. Predazzi, An Introduction to Gauge Theories and the "New Physics" (Cambridge University Press, Cambridge, England, 1982).

[27] C. Prescott, W. Atwood, R. L. Cottrell, H. DeStaebler, E. L. Garwin et al., Parity nonconservation in inelastic electron scattering, Phys. Lett. B 77, 347 (1978).

[28] C. Y. Prescott, W. B. Atwood, R. L. A. Cottrell, and H. DeStaebler et al., Further measurements of parity nonconservation in inelastic electron scattering, Phys. Lett. B 84, 524 (1979).

[29] L. M. Barkov and M. S. Zolotorev, Parity violation in atomic bismuth, Phys. Lett. B 85, 308 (1979).

[30] M. Born and E. Wolf, Principles of Optics: Electromagnetic Theory of Propagation, Interference and Diffraction of Light (Pergamon Press, New York, 1965).

[31] M. L. Goldberger and R. M. Watson, Collision Theory (Wiley, New York, 1984). 